\title{
DIRECTIONAL MULTISCALE PROCESSING OF IMAGES USING WAVELETS WITH COMPOSITE DILATIONS
}

\author{
GLENN R. EASLEY, DEMETRIO LABATE, VISHAL M. PATEL
}

\begin{abstract}
It is widely recognized that the performance of many image processing algorithms can be significantly improved by applying multiscale image representations with the ability to handle very efficiently directional and other geometric features. Wavelets with composite dilations offer a flexible and especially effective framework for the construction of such representations. Unlike traditional wavelets, this approach enables the construction of waveforms ranging not only over various scales and locations but also over various orientations and other orthogonal transformations. Several useful constructions are derived from this approach, including the well-known shearlet representation and new ones, introduced in this paper. In this work, we introduce and apply a novel multiscale image decomposition algorithm for the efficient digital implementation of wavelets with composite dilations. Due to its ability to handle geometric features efficiently, our new image processing algorithms provide consistent improvements upon competing state-of-the-art methods, as illustrated on a number of image denoising and image enhancement demonstrations.
\end{abstract}

\section{INTRODUCTION}

It has become commonly understood that while $1 \mathrm{D}$ wavelets are optimal at approximating point singularities, their $2 \mathrm{D}$ separable counterparts are not equally effective at approximating singularities along curves which model edges in images. The need for directional filtering in order to improve multidimensional data processing was early recognized, for example, in the works [1, 23, 41]. More recently, new

Key words and phrases. Wavelets, directional filter banks, wavelets with composite dilations, contourlets, shearlets, curvelets. 
generations of directional representations were developed which exhibit near optimal approximations on the class of cartoon images. The most notable of these representations are the curvelets $[42,8]$, the contourlets [13] and the shearlets [26, 34], which are constructed by defining systems of analyzing waveforms ranging not only at various scales and locations, like traditional wavelets, but also at various orientations, with the number of orientations increasing at finer scales.

Within the context of improved multidimensional representations, the theory of wavelets with composite dilations, originally developed in $[26,27,28]$, is especially important. This framework is a generalization of the classical theory from which traditional wavelets are derived, and it provides a very flexible setting for the construction of many truly multidimensional variants of wavelets, such as the well known construction of shearlets ${ }^{1}$. Several additional sophisticated constructions using this approach were obtained by Blanchard $[4,5]$, by Kryshtal and Blanchard in a paper which exploits the connection with crystallographic groups [6], and by Kryshtal et al. [32]. Furthermore, it was recently shown by two of the authors in [16] that several recent filter bank constructions such as the hybrid wavelets of Eslami and Rada $[20,21,22]$ and the variants of the contourlets construction proposed in $[31,45]$ can either be derived or are closely related to systems obtained from the framework of wavelets with composite dilations.

These observations indicate the potential of wavelets with composite dilations for the construction of directional multiscale representations going far beyond traditional wavelets with respect to their ability to represent geometric features. However, except for some very special cases, no satisfactory method for designing efficient numerical implementations of wavelets with composite dilations was developed so far, due to the difficulty of adapting the standard wavelet implementation algorithms to this more general setting. The goal of this paper is to introduce a

\footnotetext{
${ }^{1}$ Even though the shearlet system is not exactly an example of wavelets with composite dilations, it is closely related to this framework being defined as a union of two such systems [26, 34].
} 
new general procedure for the design of discrete multiscale transforms which takes full advantage of the geometric features associated with the framework of wavelets with composite dilations. Using this approach, we are able to design and implement several new classes of directional discrete transforms and to obtain improved implementations of known ones. As will be illustrated in this paper, the newly derived algorithms based on wavelets with composite dilations are highly competitive in imaging applications such as denoising and enhancement.

Recall that, for tasks such as image denoising and enhancement, where the objective is to extract or emphasize certain image features, it is often beneficial to use redundant representations. A standard method for designing nonsubsampled directional representations is to use critically sampled transformations based on 2D directional filters that satisfies Bezout's identity, as done in [12, 22]. As will become clear below, this approach is frequently associated with filters that do not faithfully match with the desired theoretical frequency decomposition. By contrast, in this paper we introduce a novel filter bank construction technique that enables the projection of the data directly onto the desired directionally-oriented frequency subbands. A key new feature of our construction is the ability to generate the transform coefficients by directly applying the action of the matrices associated with the frequency plane decomposition. This is in contrast to earlier implementations such as the discrete shearlet transform in [17], which was designed to mimic the desired frequency plane decomposition. Not only does our new approach follow directly from the theoretical setting, but it also allows for more sophisticated composite wavelet decompositions enabling a much finer handling of the geometry in the data. As special cases of our approach, we obtain an improved implementation of the shearlet transform and we introduce new hyperbolic composite wavelet transform. This last transform has potentially high impact in deconvolution and other image enhancement applications, as indicated by the novel decompositions suggested in [10] and by the techniques for dealing with motion blur recently proposed 
in [19]. This is due to the special decomposition of the frequency plane associated with this transform which provides a somewhat finer partition of the low-frequency region vs. the high-frequency one.

Finally, we wish to mention the important work by Durand [15], who explores a large family of (discrete) directional wavelets and derives their implementation from a single nonseparable filter bank structure. With respect to our approach which is continuous (i.e., we deal with functions in $L^{2}\left(\mathbb{R}^{2}\right)$ ), the work of Durand is purely discrete.

1.1. Paper Organization. The definition and basic properties of wavelets with composite dilations, along with novel constructions are presented in Section 2. The novel discrete implementations are discussed in Section 3. Numerical demonstrations illustrating the performance of the new constructions for denoising and image enhancement are presented in Section 4. Concluding remarks are given in Section 5.

\section{WAVELETS With COMPOSITE DILATIONS}

Let us introduce the notation which will be used subsequently. Given $\tau \in \mathbb{R}^{n}$, the translation operator $T_{\tau}$ on $L^{2}\left(\mathbb{R}^{n}\right)$ is defined by

$$
T_{\tau} f(x)=f(x-\tau), \quad x \in \mathbb{R}^{n}, \quad f \in L^{2}\left(\mathbb{R}^{n}\right) .
$$

For $a \in G L_{n}(\mathbb{R})$ (where $G L_{n}(\mathbb{R})$ denotes the group of invertible matrices on $\mathbb{R}^{n}$ ), the dilation operator $D_{a}$ is given by

$$
D_{a} f(x)=|\operatorname{det} a|^{-1 / 2} f\left(a^{-1} x\right) .
$$

We adopt the convention that the points $x \in \mathbb{R}^{n}$ are column vectors and the points $\xi \in \widehat{\mathbb{R}}^{n}$ (the Fourier domain) are row vectors. Hence a vector $x \in \mathbb{R}^{n}$ multiplying a matrix $a \in G L_{n}(\mathbb{R})$ on the right is a column vector and a vector $\xi \in \widehat{\mathbb{R}}^{n}$ multiplying a matrix $a \in G L_{n}(\mathbb{R})$ on the left is a row vector; that is, $a x \in \mathbb{R}^{n}$ and $\xi a \in \widehat{\mathbb{R}}^{n}$. Notice that we will denote matrices as lower-case letters, and use capital letters to 
denote sets. The Fourier transform of $f \in L^{2}\left(\mathbb{R}^{n}\right)$ is given by

$$
\hat{f}(\xi)=\int_{\mathbb{R}^{n}} f(x) e^{-2 \pi i \xi x} d x
$$

where $\xi \in \widehat{\mathbb{R}}^{n}$ and the inverse Fourier transform is

$$
\check{f}(x)=\int_{\widehat{\mathbb{R}}^{n}} f(\xi) e^{2 \pi i \xi x} d \xi .
$$

The standard wavelet systems generated by $\Psi=\left\{\psi_{1}, \ldots, \psi_{L}\right\} \subset L^{2}\left(\mathbb{R}^{n}\right)$ and $A=\left\{a^{i}: i \in \mathbb{Z}\right\}$ are the collections of functions of the form

$$
\left\{D_{a}^{j} T_{k} \psi_{m}: j \in \mathbb{Z}, k \in \mathbb{Z}^{n}, m=1, \ldots, L\right\},
$$

which form a Parseval frame for $L^{2}\left(\mathbb{R}^{n}\right)$. That is, for all $f \in L^{2}\left(\mathbb{R}^{n}\right)$, we have: ${ }^{2}$

$$
\|f\|^{2}=\sum_{j \in \mathbb{Z}} \sum_{k \in \mathbb{Z}^{n}} \sum_{m=1}^{L}\left|\left\langle f, D_{a}^{j} T_{k} \psi_{m}\right\rangle\right|^{2} .
$$

Note that the traditional wavelet systems are obtained with $a=2 I$, where $I$ is the identity matrix. That is, the dilation factor is the same for all coordinate axes.

The wavelets with composite dilations [26] overcome the limitations of standard wavelets in dealing with the geometry of multivariate functions by including a second set of dilations. Namely, they have the form

$$
\mathcal{A}_{A B}(\Psi)=\left\{D_{a} D_{b} T_{k} \Psi: k \in \mathbb{Z}^{n}, a \in A, b \in B\right\}
$$

where $A, B$ are countable subsets of $G L_{n}(\mathbb{R})$ and the matrices $b \in B$ satisfy $|\operatorname{det} b|=$ 1. As for standard wavelet systems, $\Psi=\left\{\psi_{1}, \ldots, \psi_{L}\right\} \subset L^{2}\left(\mathbb{R}^{n}\right)$ chosen so that

$$
\|f\|^{2}=\sum_{a \in A} \sum_{b \in B} \sum_{k \in \mathbb{Z}^{n}} \sum_{m=1}^{L}\left|\left\langle f, D_{a} D_{b} T_{k} \psi_{m}\right\rangle\right|^{2},
$$

\footnotetext{
${ }^{2}$ Recall that an orthonormal basis is a special case of a Parseval frame; however, the elements of a Parseval frame need not be orthogonal.
} 
for any $f \in L^{2}\left(\mathbb{R}^{n}\right)$. Usually, the matrices $a \in A$ are expanding (but not necessarily isotropic, as in the traditional wavelet case); the matrices $b \in B$, which are nonexpanding, are associated with rotations and other orthogonal transformations. As a result, one can define systems of wavelets with composite dilations containing elements that are "long and narrow" and range over many locations, scales, shapes and directions. Building on this idea [26, 34], shearlets are able to provide a nearly optimally sparse representation for a general class of images [24, 25].

The theory of wavelets with composite dilations extends nicely many of the standard results of wavelet theory (see $[26,27,28])$ and, at the same time, it allows for a much richer geometric structure. In particular, it is rather straightforward to obtain the following simple conditions for the constructions of wavelets with composite dilations in the case where the generator $\psi$ is chosen such that $\hat{\psi}=\chi_{S}$, where $S \subset \mathbb{R}^{2}$ and $\chi_{S}$ denotes the characteristic function of $S$. We state the theorem in dimension $n=2$, but it holds in any dimension. Notice that, since the set $S$ is defined in the Fourier domain, matrices act on it from the right, according to our general convention stated above.

Theorem 1 ([27]). Let $A, B$ be subsets of $G L_{2}(\mathbb{R})$ and $S \subset F \subset \mathbb{R}^{2}$. Suppose that the following conditions hold:

(1) $\widehat{\mathbb{R}}^{2}=\bigcup_{k \in \mathbb{Z}^{2}}(F+k)$,

(2) $\widehat{\mathbb{R}}^{2}=\bigcup_{a \in A, b \in B} S(a b)^{-1}$,

where the unions are disjoint up to a set of measure zero. Then for $\psi$ such that $\hat{\psi}=\chi_{S}$ the system $\mathcal{A}_{A B}(\psi)$ is a Parseval frame for $L^{2}\left(\mathbb{R}^{2}\right)$. If, in addition, $\|\psi\|=$ 1 , then $\mathcal{A}_{A B}$ is an orthonormal basis for $L^{2}\left(\mathbb{R}^{2}\right)$.

Hince, similar to the wavelet case, $\mathcal{A}_{A B}$ is Parseval frame if the collections of $A$ and $B$-dilations of $S$ form a tiling of the frequency plane. 
It is clear that the systems described in Theorem 1 are not well-localized in the spatial domain, since their elements are characteristic functions of sets in the frequency domain and, hence, have slow spatial decay. Wavelets with composite dilations which are well-localized require ad hoc constructions such as the shearlets in [24] or the constructions described in [16]. Some new well-localized constructions will be introduced in this paper.

2.1. Some Constructions. In this section, we apply Theorem 1 to construct several examples of wavelets with composite dilations for $L^{2}\left(\mathbb{R}^{2}\right)$. This is useful both to illustrate the variety of possible constructions which can be derived from this approach, and to set the groundwork for new discrete multiscale transforms which will be developed in Section 3. Note that Constructions 1-3 are not new (see [27, 28]), and are included for comparison. As indicated below, Constructions 1-2 are formally equivalent to systems recently introduced in the literature using ad hoc constructions, but can be derived from the general framework of wavelets with composite dilations. Construction 4 is new.

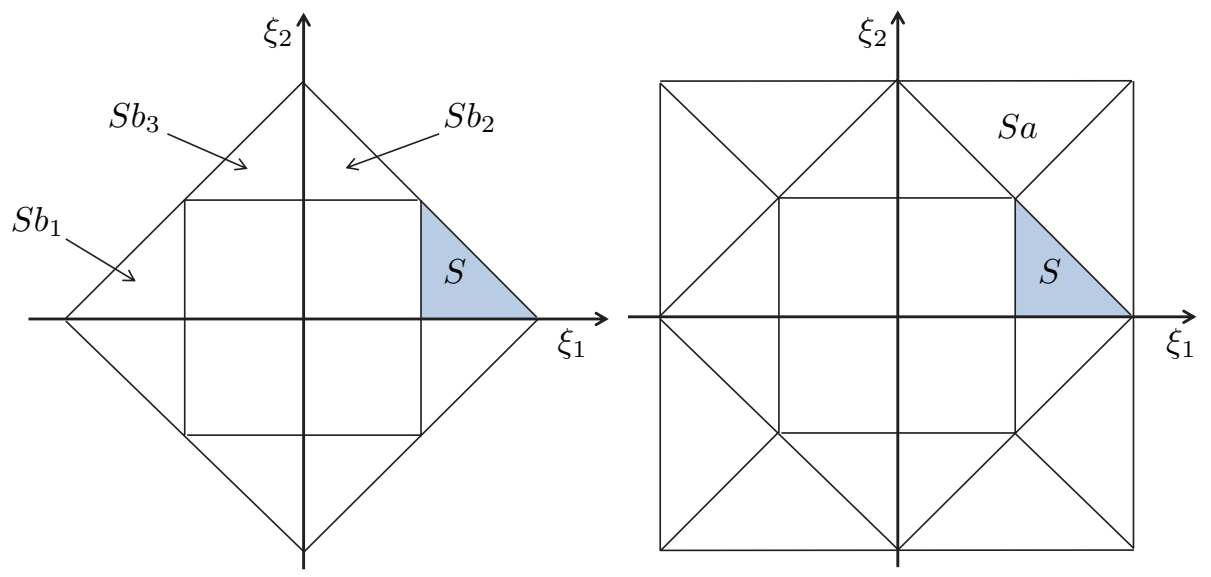

FiguRE 1. Tiling of the frequency domain associated with a system of wavelets with composite dilations, where $a$ is the quincunx dilation matrix and $B$ is the group of symmetries of the square. Notices that, since $S \in \widehat{\mathbb{R}}^{2}$, the matrices $a$ and $b_{\ell}$ acts on it from the right, according to our convention. 
2.1.1. Construction 1. Let $A=\left\{a^{j}: j \in \mathbb{Z}\right\}$ where $a=\left(\begin{array}{cc}1 & 1 \\ -1 & 1\end{array}\right)$ (the quincunx matrix) and let $B$ be the group of symmetries of the square, that is, $B=\left\{ \pm b_{0}, \pm b_{1}, \pm b_{2}, \pm b_{3}\right\}$ where $b_{0}=\left(\begin{array}{ll}1 & 0 \\ 0 & 1\end{array}\right), b_{1}=\left(\begin{array}{cc}1 & 0 \\ 0 & -1\end{array}\right), b_{2}=\left(\begin{array}{ll}0 & 1 \\ 1 & 0\end{array}\right), b_{3}=\left(\begin{array}{cc}0 & -1 \\ -1 & 0\end{array}\right)$. Let $\hat{\psi}(\xi)=\chi_{S}(\xi)$, where the set $S$ is the union of the triangles with vertices $(1,0),(2,0),(1,1)$ and $(-1,0),(-2,0),(-1,-1)$, which is illustrated in Figure 1. A direct calculation shows that $S$ satisfies the assumptions of Theorem 1, so that the system

$$
\mathcal{A}_{A B}(\psi)=\left\{D_{a}^{j} D_{b} T_{k} \psi: j \in \mathbb{Z}, b \in B, k \in \mathbb{Z}^{2}\right\}
$$

is an orthonormal basis for $L^{2}\left(\mathbb{R}^{2}\right)$ (in fact, it is a Parseval frame and $\|\psi\|=1$ ).

As noticed in [16], the frequency partition achieved by the Hybrid Quincunx Wavelet Directional Transform (HQWDT) from [22] is a simple modification of this construction, which is obtained by splitting each triangle of the set $S$ into 2 smaller triangles. Another example, with the same matrices $A$ and $B$, but a different generating set $S$, is illustrated in Figure 2 on the left. For future labeling, we will refer to this second construction concisely as the ab-star decomposition alluding to its star-like frequency tiling and its composite wavelet origin.

2.1.2. Construction 2. Let $A=\left\{a^{j}: j \in \mathbb{Z}\right\}$ where $a=\left(\begin{array}{ll}2 & 0 \\ 0 & 2\end{array}\right)$ and consider $B=$ $\left\{b_{0}, b_{1}, b_{2}, b_{3}\right\}$ where $b_{0}=\left(\begin{array}{ll}1 & 0 \\ 0 & 1\end{array}\right), b_{1}=\left(\begin{array}{cc}1 & 0 \\ 0 & -1\end{array}\right), b_{2}=\left(\begin{array}{ll}0 & 1 \\ 1 & 0\end{array}\right), b_{3}=\left(\begin{array}{cc}0 & -1 \\ -1 & 0\end{array}\right)$. Let $R$ be the union of the trapezoid with vertices $(1,0),(2,0),(1,1),(2,2)$ and the symmetric one with vertices $(-1,0),(-2,0),(-1,-1),(-2,-2)$. Next, we partition each trapezoid into right triangles $R_{m}, m=1,2,3$, as illustrated in Figure 2 on the right. Hence we define $\Psi=\left\{\psi^{m}: m=1,2,3\right\}$, where $\hat{\psi}^{m}(\xi)=\chi_{R_{m}}(\xi)$. Then the system

$$
\mathcal{A}_{A B}(\Psi)=\left\{D_{a}^{j} D_{b} T_{k} \Psi: j \in \mathbb{Z}, b \in B, k \in \mathbb{Z}^{2}\right\}
$$

is an orthonormal basis for $L^{2}\left(\mathbb{R}^{2}\right)$. 



FIGURE 2. Left: tiling of the frequency domain associated with a system of wavelets with composite dilations where $a$ is the quincunx dilation matrix and $B$ is the group of symmetries of the square. While $a$ and $B$ are as in Fig. 1, the generator $\psi$ is different. Right: tiling of the frequency domain associated with a system of wavelets with composite dilations where $a=2 I$ and $B$ is the group of symmetries of the square.

2.1.3. Construction 3. Another example of a system of wavelets with composite dilations is obtained by keeping the same set $A$ of dilation matrices as in Construction 2 , and redefining $B$ as the set $\left\{b^{\ell}:-3 \leq \ell \leq 2\right\}$, where $b$ is the shear matrix $\left(\begin{array}{ll}1 & 1 \\ 0 & 1\end{array}\right)$. For that, let $R$ be the union of the trapezoid with vertices $(1,0),(2,0)$, $(1,1 / 3),(2,2 / 3)$ and the symmetric one with vertices $(-1,0),(-2,0),(-1,-1 / 3)$, $(-2,-2 / 3), \tilde{R}=R r$, where $r=\left(\begin{array}{cc}0 & 1 \\ -1 & 0\end{array}\right)$ and define $\Psi=\left\{\psi^{m}: m=1,2\right\}$, where $\hat{\psi}^{1}=\chi_{R}$, and $\hat{\psi}^{2}=\chi_{\tilde{R}}$. It follows that the system

$$
\mathcal{A}_{A B}(\Psi)=\left\{D_{a}^{j} D_{b} T_{k} \Psi: j \in \mathbb{Z}, b \in B, k \in \mathbb{Z}^{2}\right\}
$$

is an orthonormal basis for $L^{2}\left(\mathbb{R}^{2}\right)$. For practical applications, it is useful to modify this system by restricting the scale parameter to the values $j \geq 0$ and by taking care of the low frequency region of the frequency plane using a standard wavelet basis. The frequency tiling corresponding to this system is illustrated in Figure 3. As observed in [16], this frequency tiling is similar to the one used for the NonUniform Directional Filter Bank (NUDFB) in [38] and to a construction in [14]. If 


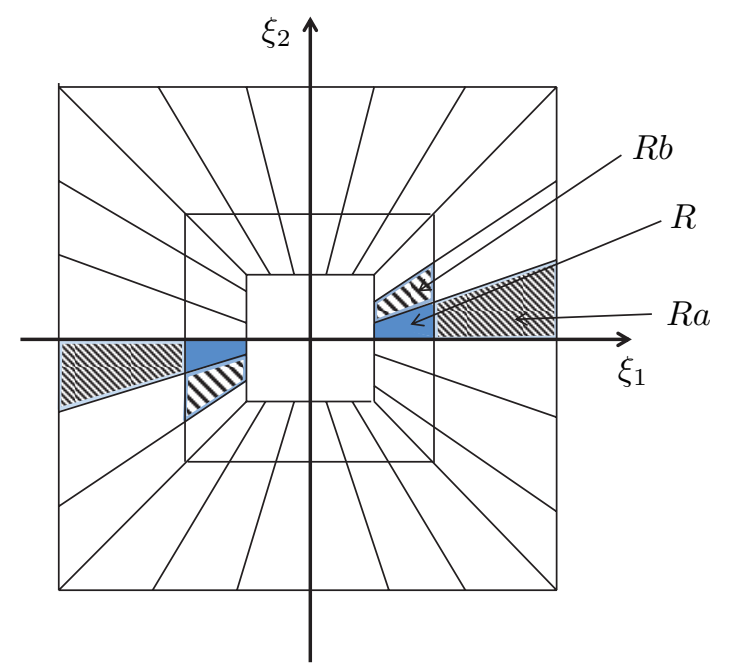

FiguRE 3. Tiling of the frequency domain associated with a system of wavelets with composite dilations where $a=2 I$ and $B$ is a set of shearing matrices.

this construction is combined with a separable generator, then one obtains the frequency tiling corresponding to the Hybrid Wavelet Directional Transform (HWDT) from [22] and to the directional filter bank constructions found in [15, 36, 45].

\subsection{Construction 4. Hyperbolic tiling. ${ }^{3}$}

Another example of a system of wavelets with composite dilations is obtained by using sets of matrices of the form

(1) $B=\left\{b^{\ell}=\left(\begin{array}{cc}1 / \lambda & 0 \\ 0 & \lambda\end{array}\right)^{\ell}: \ell \in \mathbb{Z}\right\}, \quad A=\left\{a^{j}=\left(\begin{array}{cc}\sqrt{2} & 0 \\ 0 & \sqrt{2}\end{array}\right)^{j}: j \in \mathbb{Z}\right\}$,

where $\lambda>1$ is a fixed parameter. In the following, we will set $\lambda=\sqrt{2}$, but the discussion below can be easily extended to other choices of $\lambda$.

Let us start by noticing that, for each $k>0$, the set $H_{k}=\left\{\left(\xi_{1}, \xi_{2}\right) \in \widehat{\mathbb{R}}^{2}: \xi_{1} \xi_{2}=\right.$ $k\}$ consists of two branches of hyperbolas. For any $\xi=\left(\xi_{1}, \xi_{2}\right) \in H_{k}$, every other point $\xi^{\prime}$ on the same branch of the hyperbola has the unique representation $\xi^{\prime}=$ $\left(\xi_{1} \gamma^{-t}, \xi_{2} \gamma^{t}\right)$ for some $t \in \mathbb{R}$, where $\gamma>1$ is fixed. In particular, any $\xi=\left(\xi_{1}, \xi_{2}\right)$ in

\footnotetext{
${ }^{3}$ Some ideas of this construction were introduced by one of the authors and collaborators in [27].
} 

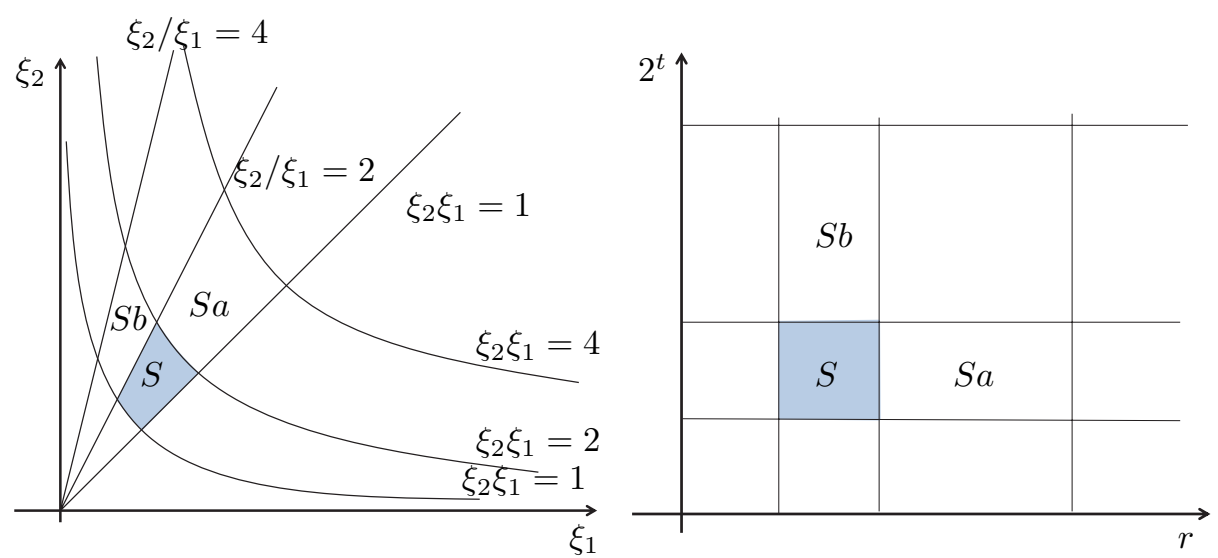

FIGURE 4. The figure on the left shows the hyperbolic trapezoid $S=S_{I}$, in quadrant $I$, and the action of the matrices $a$ and $b$ on it. The plot on the right shows the same regions in the coordinate system defined by $r$ and $2^{t}$.

quadrant I can be parametrized by

$$
\xi(r, t)=\left(\sqrt{r}(\sqrt{2})^{-t}, \sqrt{r}(\sqrt{2})^{t}\right),
$$

where $r \geq 0, t \in \mathbb{R}$. This implies that $r=\xi_{1} \xi_{2}$ and $2^{t}=\frac{\xi_{2}}{\xi_{1}}$. For any $k_{1}<k_{2}$, a set $\left\{\xi(r, t): k_{1} \leq r<k_{2}\right\}$ is a hyperbolic strip and, for $m_{1}<m_{2}$, a set $\left\{\xi(r, t): k_{1} \leq\right.$ $\left.r<k_{2}, m_{1} \leq 2^{t} \leq m_{2}\right\}$ is a hyperbolic trapezoid. We have the following observation.

Proposition 2. Let $S_{I}$ be the hyperbolic trapezoid $S_{I}=\{\xi(r, t): 1 \leq r<2,1 \leq$

$$
\begin{array}{r}
\left.2^{t} \leq 2\right\}, a=\left(\begin{array}{cc}
\sqrt{2} & 0 \\
0 & \sqrt{2}
\end{array}\right) \text { and } b=\left(\begin{array}{cc}
1 / \sqrt{2} & 0 \\
0 & \sqrt{2}
\end{array}\right) . \text { Then } \\
\bigcup_{j \in \mathbb{Z}} \bigcup_{\ell \in \mathbb{Z}} S_{I} a^{j} b^{\ell}=\text { quadrant } I,
\end{array}
$$

where the union is disjoint, up to sets of measure zero.

Proof. For any $k \neq 0$, the matrices $b^{\ell}, \ell \in \mathbb{Z}$, preserve the hyperbolas $H_{k}$ since

$$
\xi b^{\ell}=\left(\xi_{1}, \xi_{2}\right)\left(\begin{array}{cc}
(\sqrt{2})^{-\ell} & 0 \\
0 & (\sqrt{2})^{\ell}
\end{array}\right)=\left(\xi_{1}(\sqrt{2})^{-\ell}, \xi_{2}(\sqrt{2})^{\ell}\right)=\left(\eta_{1}, \eta_{2}\right)
$$


and $\eta_{1} \eta_{2}=\xi_{1} \xi_{2}$. Hence, the right action of $b^{\ell}, \ell \in \mathbb{Z}$, maps an hyperbolic strip into itself (see Figure 4). In addition, a direct calculation shows that, if $\xi_{2}=m \xi_{1}$ and $\left(\eta_{1}, \eta_{2}\right)=\left(\xi_{1}, \xi_{2}\right) b^{\ell}$, then $\frac{\eta_{2}}{\eta_{1}}=2^{\ell} \frac{\xi_{2}}{\xi_{1}}=2^{\ell} m$. It follows that $b^{\ell}, \ell \in \mathbb{Z}$, maps a line through the origin of slope $m$ into a line through the origin of slope $2^{\ell} m$. From the observations above, it follows that the hyperbolic trapezoid $S_{I}$ is a tiling set of the hyperbolic strip $\{\xi(r, t): 1 \leq r<2\}$ for the dilations $b^{\ell}, \ell \in \mathbb{Z}$.

Next observe that $a$ maps the hyperbola $\xi_{1} \xi_{2}=k$ to the hyperbola $\xi_{1} \xi_{2}=2 k$, and that $a^{j}$ maps the hyperbolic strip $\{\xi(r, t): 1 \leq r<2\}$ to the hyperbolic strip $\left\{\xi(r, t): 2^{j} \leq r<2^{j+1}\right\}$ (see Figure 4). The proof follows by combining this observation with the previous one about the action of the dilations $b^{\ell}$ on $S_{I}$.
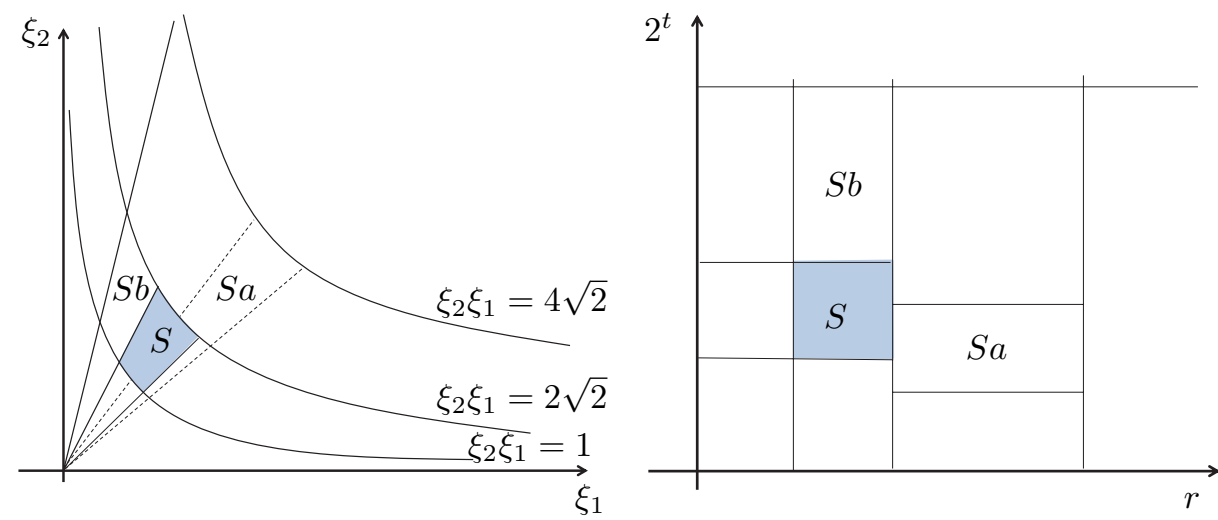

FIGURE 5. The plot on the left shows the hyperbolic trapezoid $S=$ $S_{I}^{\prime}$, in quadrant $I$, and the action of the matrices $a$ and $b$ on it. The plot on the right shows the same regions in the coordinate system defined by $r$ and $2^{t}$.

To obtain a tiling of the whole frequency plane, let $S_{I I I}$ be the symmetric extension of $S_{I}$ in quadrant III, i.e., $S_{I I I}=\left\{x \in \mathbb{R}^{2}:-x \in S\right\}$; in quadrant IV, define

$$
S_{I V}=\left\{\left(\xi_{1}, \xi_{2}\right):\left(-\xi_{1}, \xi_{2}\right) \in S_{I}\right\}
$$


and its symmetric extension $S_{I I}$ in quadrant II. It follows that

$$
\bigcup_{j \in \mathbb{Z}} \bigcup_{\ell \in \mathbb{Z}}\left(S_{I} \cup S_{I I} \cup S_{I I I} \cup S_{I V}\right) a^{j} b^{\ell}=\mathbb{R}^{2}
$$

where again the union is disjoint, up to sets of measure zero. Hence, letting $\Psi=$ $\left(\psi_{I}, \psi_{I I}, \psi_{I I I}, \psi_{I V}\right)$, where $\hat{\psi}_{I}=\chi_{S_{I}}, \ldots, \hat{\psi}_{I V}=\chi_{S_{I V}}$, it follows from Theorem 1 that the system of wavelets with composite dilations

$$
\mathcal{A}_{A B}(\Psi)=\left\{D_{a}^{j} D_{b}^{\ell} T_{k} \Psi: j \in \mathbb{Z}, k \in \mathbb{Z}^{2}, \ell \in \mathbb{Z}\right\}
$$

is a Parseval frame of $L^{2}\left(\mathbb{R}^{2}\right)$.

A variant of the construction above is obtained by replacing the set of diagonal matrices $A$ in (1) with the set

$$
A=\left\{a^{j}=\left(\begin{array}{cc}
2 & 0 \\
0 & \sqrt{2}
\end{array}\right)^{j}: j \in \mathbb{Z}\right\} .
$$

These matrices produce parabolic scaling, that it, the dilation factor along one orthogonal axis is quadratic with respect to the other axis. This scaling factor plays a critical role in the sparsity properties of curvelets and shearlets [8, 24, 33].

Similar to the construction above, let $\widetilde{S}_{I}$ be the hyperbolic trapezoid in the frequency plane defined by

$$
\widetilde{S}_{I}=\left\{\xi(r, t): \sqrt{2} / 2 \leq r<2,1 \leq 2^{t} \leq 2\right\}
$$

Again, the right action of $B$ maps any hyperbolic strip into itself. The matrix $a \in A$ maps the hyperbola $\xi_{1} \xi_{2}=k$ to the hyperbola $\xi_{1} \xi_{2}=2 \sqrt{2} k$ and, thus, $a^{j}$ maps the hyperbolic strip $\{\xi(r, t): \sqrt{2} / 2 \leq r<2\}$ to the hyperbolic strip $\left\{\xi(r, t): 2(2 \sqrt{2})^{j-1} \leq r<2(2 \sqrt{2})^{j}\right\}$. Unlike the previous construction, however, the matrix $a \in A$ does not preserve lines through the origin since it maps a line $\xi_{2}=m \xi_{1}$ into $\xi_{2}^{\prime}=\frac{\sqrt{2}}{2} m \xi_{1}^{\prime}$. The action of the matrices $a$ and $b$ on $\widetilde{S}_{I}$ is shown in Figure 5, illustrating that the sets $S_{I}^{\prime} a^{i} b^{\ell}$ become increasingly more elongated as 
$i \rightarrow \infty$. Similar to the previous construction, by defining trapezoids $\widetilde{S}_{I I}, \widetilde{S}_{I I I}$ and $\widetilde{S}_{I V}$ in the other quadrants, it is easy to verify that the tiling property holds:

$$
\bigcup_{j \in \mathbb{Z}} \bigcup_{\ell \in \mathbb{Z}}\left(\widetilde{S}_{I} \cup \widetilde{S}_{I I} \cup \widetilde{S}_{I I I} \cup \widetilde{S}_{I V}\right) a^{j} b^{\ell}=\mathbb{R}^{2} .
$$

Thus, letting $\Psi=\left(\psi_{I}, \psi_{I I}, \psi_{I I I}, \psi_{I V}\right)$, where $\hat{\psi}_{I}=\chi_{\widetilde{S}_{I}}, \ldots, \hat{\psi}_{I V}=\chi_{\widetilde{S}_{I V}}$, it follows from Theorem 1 that the system of wavelets with composite dilations

$$
\mathcal{A}_{A B}(\Psi)=\left\{D_{a}^{j} D_{b}^{\ell} T_{k} \Psi: j \in \mathbb{Z}, k \in \mathbb{Z}^{2}, \ell \in \mathbb{Z}\right\}
$$

is a Parseval frame of $L^{2}\left(\mathbb{R}^{2}\right)$.

2.2.1. Well-localized Construction. In order to construct hyperbolic systems of wavelets with composite dilations which are well-localized, we recall the following result.

Theorem 3 ([27]). Let $\psi \in L^{2}\left(\mathbb{R}^{2}\right)$ be such that $\operatorname{supp} \hat{\psi} \subset Q=[-1 / 2,1 / 2]^{2}$, and

$$
\sum_{j, \ell \in \mathbb{Z}}\left|\hat{\psi}\left(\xi a^{j} b^{\ell}\right)\right|^{2}=1 \quad \text { a.e. } \xi \in \widehat{\mathbb{R}}^{2},
$$

where $a, b \in G L_{2}(\mathbb{R})$. Then the system of wavelets with composite dilations (1), where $A=\left\{a^{j}: j \in \mathbb{Z}\right\}$ and $B=\left\{b^{\ell}: \ell \in \mathbb{Z}\right\}$, is a Parseval frame of $L^{2}\left(\mathbb{R}^{2}\right)$.

For $\xi=\left(\xi_{1}, \xi_{2}\right) \in \widehat{\mathbb{R}}^{2}$, with $\xi_{1} \neq 0$, let $\hat{\psi}$ be defined by

$$
\hat{\psi}\left(\xi_{1}, \xi_{2}\right)=V\left(\xi_{1} \xi_{2}\right) W\left(\frac{\xi_{2}}{\xi_{1}}\right)
$$

where $V, W \in C_{c}^{\infty}(\mathbb{R})$ satisfy $\operatorname{supp} V \subset\left[\frac{1}{88}, \frac{1}{11}\right]$, supp $W \subset\left[\frac{2}{3}, \frac{8}{3}\right]$,

$$
\sum_{j \in \mathbb{Z}}\left|V\left(2^{j} r\right)\right|^{2}=1 \quad \text { for a.e. } r \geq 0
$$

and

$$
\sum_{\ell \in \mathbb{Z}}\left|W\left(2^{\ell} 2^{t}\right)\right|^{2}=1 \quad \text { for a.e. } t \in \mathbb{R} \text {. }
$$


The functions $V$ and $W$ can be obtained by appropriately rescaling a Meyer wavelet and restricting its domain to the positive axis in the Fourier domain.

Hence we have the following corollary of Theorem 3.

Proposition 4. Let $\psi \in L^{2}\left(\mathbb{R}^{2}\right)$ be given by $(3)$ and $\psi^{\prime}$ be defined by $\hat{\psi}^{\prime}\left(\xi_{1}, \xi_{2}\right)=$ $\hat{\psi}\left(-\xi_{1}, \xi_{2}\right)$. Then, for $A$ and $B$ given by (1) and $\Psi=\left\{\psi, \psi^{\prime}\right\}$, the system of wavelets with composite dilations

$$
\mathcal{A}_{A B}(\Psi)=\left\{D_{a}^{j} D_{b}^{\ell} T_{k} \Psi: j \in \mathbb{Z}, k \in \mathbb{Z}^{2}, \ell \in \mathbb{Z}\right\}
$$

is a Parseval frame of $L^{2}\left(\mathbb{R}^{2}\right)$.

Proof. The support conditions on $V, W$ ensure that supp $\hat{\psi}$ is a pair of trapezoidal regions in quadrants I and III which are contained inside the unit cube $[-1 / 2,1 / 2]^{2}$. In fact, $V$ is constant along each branch of the hyperbola and is defined along lines through the origin. Its support is contained between the hyperbolas $\xi_{1} \xi_{2}=\frac{1}{88}$ and $\xi_{1} \xi_{2}=\frac{1}{11} . W$ is constant along each line through the origin, is defined along hyperbolas and its support is contained in the cone defined by the lines through the origin of slopes $\xi_{2}=\frac{2}{3} \xi_{1}$ and $\xi_{2}=\frac{8}{3} \xi_{1}$. In addition, a direct calculation shows that, for all $\xi=\left(\xi_{1}, \xi_{2}\right)$, with $\xi_{1} \xi_{2} \geq 0$ and $\xi_{1} \neq 0$, we have:

$$
\begin{aligned}
& \sum_{j \in \mathbb{Z}} \sum_{\ell \in \mathbb{Z}}\left|\hat{\psi}\left(\xi a^{j} b^{\ell}\right)\right|^{2} \\
& =\sum_{j \in \mathbb{Z}} \sum_{\ell \in \mathbb{Z}}\left|\hat{V}\left(2^{j} \xi_{1} \xi_{2}\right)\right|^{2}\left|W\left(2^{\ell} \frac{\xi_{2}}{\xi_{1}}\right)\right|^{2} \\
& =\sum_{j \in \mathbb{Z}}\left|\hat{V}\left(2^{j} \xi_{1} \xi_{2}\right)\right|^{2} \sum_{\ell \in \mathbb{Z}}\left|W\left(2^{\ell} \frac{\xi_{2}}{\xi_{1}}\right)\right|^{2}=1,
\end{aligned}
$$

for $\xi$ in quadrants I and III in $\mathbb{R}^{2}$. A similar calculation with $\hat{\psi}$ replaced by $\hat{\psi}^{\prime}$ yields a similar result valid for all $\xi$ in quadrants II and IV. Hence $\sum_{j \in \mathbb{Z}} \sum_{\ell \in \mathbb{Z}}\left|\hat{\Psi}\left(\xi a^{j} b^{\ell}\right)\right|^{2}=$ 1, for a.e. $\xi \in \mathbb{R}^{2}$ and the proof follows from Theorem 3 . 
An argument similar to Proposition 4 shows that it is possible to construct a similar well-localized Parseval frame by replacing the set of isotropic dilations $A$ given by (1) with the anisotropic dilations (2).

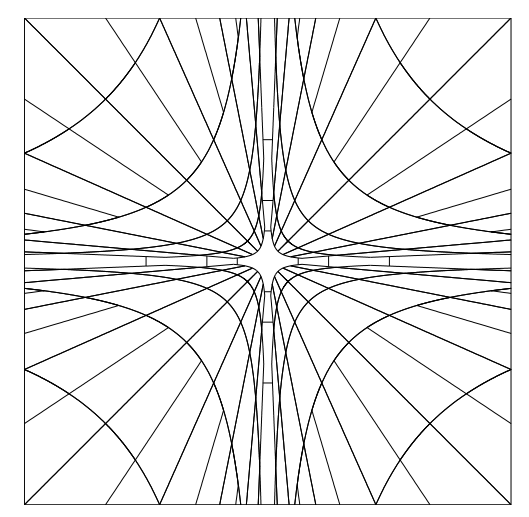

FIGURE 6. Tiling of the frequency domain associated with a hyperbolic system of wavelets with composite dilations.

Note that, for a discrete implementation of the hyperbolic systems, the indices $j$ and $\ell$ need to be limited to a finite range. The asymptotic regions not covered because of this discretization can then be dealt with by partitioning the complementary regions with a Laplacian Pyramid-type of filtering. A form of the tiling of the frequency plane associated with this construction is illustrated in Figure 6 . For the correct interpretations of this figure, observe that the elements of the welllocalized system of wavelets with composite dilations $\psi\left(a^{j} b^{\ell} x\right)$ do overlap in the frequency domain. That is, Figure 6 should be interpreted as a picture of the essential frequency support (i.e., the regions where most of the norm is concentrated), rather than the exact frequency support of the elements of the system.

2.2.2. Cone-based hyperbolic construction. The hyperbolic construction suffers from a bias in directional sensitivity along the orientations $\pm \pi / 4, \pm 3 \pi / 4$. In addition, the regions along the orthogonal axes in the frequency domain are only covered asymptotically, for values $\ell \rightarrow \pm \infty$. To overcome this situation, we define the 


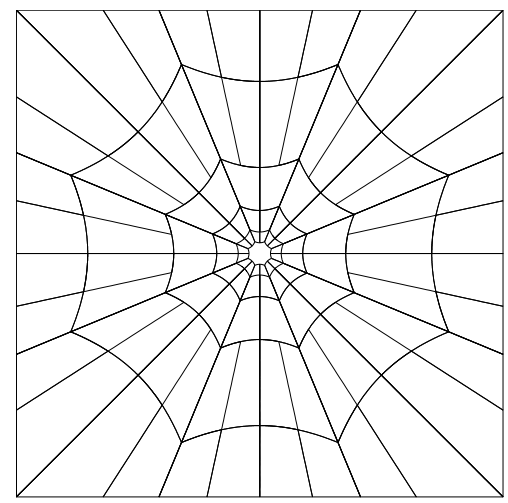

FIGURE 7. Frequency tiling of a cone-based hyperbolic composite wavelet system. Since the scaling matrix is isotropic, the number of directional subbands is fixed at each scale. If the scaling matrix is replaced by an anisotropic one, e.g., a parabolic scaling matrix, then the number of directional subbands will double at each scale.

"cone-based" system of wavelets of composite dilations

$$
\left\{D_{a}^{j} D_{b}^{\ell} T_{k} \psi: j \geq 0,|\ell| \leq L, k \in \mathbb{Z}^{2}\right\}
$$

where $b^{\ell} \in B$ given by (1), a can be the isotropic or anisotropic dilation matrix, $L$ is chosen so that the system tiles the frequency plane only in the cone $\frac{\pi}{8} \leq \frac{\xi_{2}}{\xi_{1}} \leq \frac{3 \pi}{8}$, and $\psi$ is given by (3). Using $\psi^{\prime}$ defined as in Proposition 4 one covers the frequency plane in the cone $\frac{5 \pi}{8} \leq \frac{\xi_{2}}{\xi_{1}} \leq \frac{7 \pi}{8}$. Finally, the remaining two cones in the frequency domain are covered by rotating the above systems by $\pi / 4$. The picture of the frequency tiling associated with this decomposition is given in Figure 7.

Unfortunately, the projection into cones has the effect of destroying the good localization properties of those elements of the system that overlap the cone boundaries in the Fourier domain. The analytic solution of this problem is not trivial and is beyond the scope of this paper. However, this limitation has not caused any significant loss of performance for its implementation. 


\section{Composite Wavelet Implementation}

3.1. Analysis Filter Design. In this section, we describe a novel approach for the construction of filters that match the frequency tiling associated with the desired system of wavelets with composite dilations. Given a system of wavelets with composite dilations $\mathcal{A}_{A B}(\Psi)$, this approach allows us to directly apply the set of matrices $A$ and $B$ to generate the specific frequency tiling associated with the system $\mathcal{A}_{A B}(\Psi)$.


FiguRE 8. Illustrations of filter constructions where the number of samples used is small for the purpose of presentation. The images on the left are the sequences of points $\left\{\left(\xi_{1}^{n}, \xi_{2}^{n}\right)\right\}_{n=1}^{N}$ contained in the region $S$. The images on the right are the sequences of points $\left\{\left(\bar{\eta}_{1}^{n}, \bar{\eta}_{2}^{n}\right)\right\}_{n=1}^{N}$ where $\left(\bar{\eta}_{1}^{n}, \bar{\eta}_{2}^{n}\right)=\left\lceil\left(\xi_{1}^{n}, \xi_{2}^{n}\right) a^{j} b^{\ell}\right\rceil$.

To describe our approach, consider $\hat{\psi}(\xi)=\chi_{S}(\xi)$, where $S$ corresponds to a bounded region satisfying the conditions of Theorem 1. For example, $S$ can be chosen to be a trapezoidal hyperbola and the set of matrices $A$ and $B$ to be $A=$ $\left\{a^{j}\right\}, B=\left\{b^{\ell}\right\}$, as in Construction 4 .

Hence, given a sequence of points $\left\{\left(\xi_{1}^{n}, \xi_{2}^{n}\right)\right\}_{n=1}^{N} \in S$ that densely fills the set $S$, we define $\left(\bar{\xi}_{1}^{n}, \bar{\xi}_{2}^{n}\right)=\left\lceil\left(\xi_{1}^{n}, \xi_{2}^{n}\right)\right\rceil=\left(\left\lceil\xi_{1}^{n}\right\rceil,\left\lceil\xi_{2}^{n}\right\rceil\right)$, where $\lceil\cdot\rceil$ denotes the ceiling function. To digitally create the desired filters, we assign the non-zero entries of 

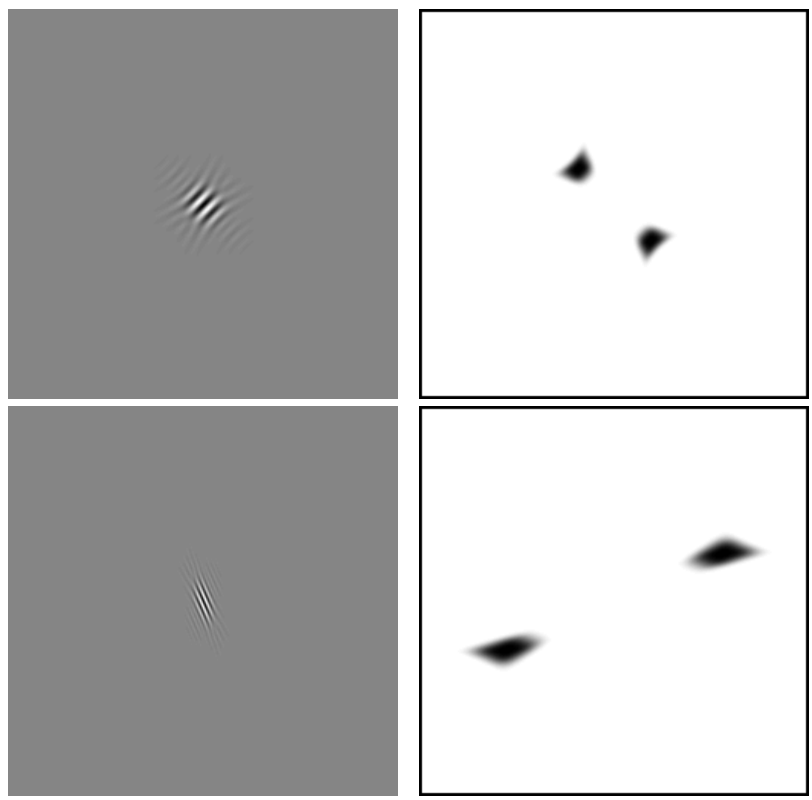

Figure 9. Examples of hyperbolic filters. From left to right: Time Domain, Frequency Domain

our starting filter $\widehat{G}_{0,0}$ by the evaluation $\widehat{G}_{0,0}\left(\bar{\xi}_{1}^{n}, \bar{\xi}_{2}^{n}\right)=1$. We then proceed to create the other filters $\left\{\widehat{G}_{i, \ell}\right\}$ by assigning the non-zeros entries as $\widehat{G}_{i, \ell}\left(\bar{\eta}_{1}^{n}, \bar{\eta}_{2}^{n}\right)=1$ for $\left(\bar{\eta}_{1}^{n}, \bar{\eta}_{2}^{n}\right)=\left\lceil\left(\xi_{1}^{n}, \xi_{2}^{n}\right) a^{j} b^{\ell}\right\rceil$. Note that $N$ needs to be chosen large enough so that the points $\left(\bar{\eta}_{1}^{n}, \bar{\eta}_{2}^{n}\right)$ are dense enough to fill out the regions $S_{j, \ell}=S a^{j} b^{\ell}$ completely in terms of its pixelated image for all desired values of $j$ and $\ell$. Thus, the density is relative to the size of the pixelated image grid. An example of such an $N$ is provided in the appendix. Figure 8 illustrates this construction.

This construction can be modified to create the well-localized version of wavelets of composite dilations by the following modification. We start by creating an initial densely supported filter with the desired windowing. By keeping track of the multiple assigned grid points, the windowing can be appropriately compensated by assigning the average windowed value at these point locations. Examples of this construction are shown in Figure 9 and a pseudo-code for creating such filters is given in the Appendix. This construction process can be applied to any planar 
region and can be viewed as a refinement of the original discrete shearlet transform construction given in [17]. In fact, when the techniques suggested in this paper are used to design filters for a revised discrete shearlet transform, the newly constructed discrete transform performs significantly better than its first incarnation. Recall that the original shearlet implementation was based on the use of a mapping function that performed a re-arrangement of windowed data in a pseudo-polar grid onto a Cartesian grid. In our new implementation, we avoid the use of the mapping function and are able to produce the appropriate windowed data directly onto the Cartesian grid. In addition, this implementation is multi-channel, so that the estimates provided from individual filtered coefficients are not level-dependent and this improves the transform's conditioning. In contrast with [17], where an inverse mapping function was used to take care of weighting the multiple assigned pixel values, by avoiding the re-mapping process, we can now avoid creating rearrangement domains (a process which usually generated some artifacts). In fact, we are able to obtain the desired filters by directly applying the $A$ and $B$ dilations associated with the desired composite wavelet decompositions. This way, the discrete implementation provides a perfect match with its theoretical counterpart and it allows one to deal even with very complicated geometrical decompositions in the frequency plane. See Figure 10 for a representative illustration of the filters associated with the new implementation.

3.2. Synthesis Filter Design. It would seem that our construction method of analysis filters should be extendible for the creation of the synthesis filters. However, by dealing with the non-uniformity caused by the discretization of a continuous formulation, we have introduced slight imperfections in the windowing. These slight deviations can be particularly unforgiving in a multi-channel based implementation. Consider, as an example, reformulating the contourlet transform naively into a multi-channel implementation by applying the transform to a delta function to find the analysis and synthesis filters. Figure 11 gives a visual illustration of how 




Figure 10. An illustration of a multi-channel filter bank designed to implement wavelets with composite dilations.

this approach fails to achieve perfect reconstruction. This is particularly interesting since the standard implementation of contourlets achieves perfect reconstruction.

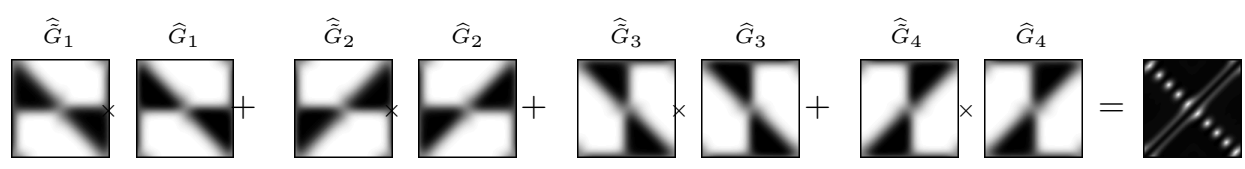

FIGURE 11. By applying the nonsubsampled contourlet transform to a delta function, we can obtain directional filters. Failure of the perfect reconstruction condition is depicted using the analysis and synthesis filters found by applying the 2nd level decomposition of contourlets to a delta function.

To improve upon the reconstruction estimate, we will construct the synthesis filters by using a method devised for solving a related problem known as the Multichannel Deconvolution Problem (MDP) which can roughly be stated as follows.

Given a collection $\left\{G_{i}\right\}_{i=1}^{m}$ of distributions on $\mathbb{R}^{d}(d \geq 2)$, find a collection $\left\{\tilde{G}_{j}\right\}_{j=1}^{m}$ 
of distributions such that

$$
\sum_{j=1}^{m} \tilde{G}_{j} * G_{j}=\delta
$$

where $\delta$ is a Dirac delta distribution. In the Fourier-Laplace domain, when the distributions are assumed to be compactly supported, this equation is referred to as the analytic Bezout equation. This problem has a connection with the polynomial Bezout equation which is usually solved for computing the filters associated with traditional filter banks (see [11] for more details).

Several methods for solving the MDP in a discrete setting provide a way of constructing appropriate synthesis filters (see [39, 40, 2, 3, 29, 18, 11, 48] for details on some of these methods). One of the earliest and simplest methods for solving this problem was given in [39]. To explain its derivation, we formulate the problem in the Fourier domain as follows. Suppose we wish to recover the image $f$ and that we are given $m$ blurred images $s_{j}$, i.e.

$$
\widehat{s_{j}}\left(\xi_{1}, \xi_{2}\right)=\widehat{f}\left(\xi_{1}, \xi_{2}\right) \widehat{G_{j}}\left(\xi_{1}, \xi_{2}\right)+\widehat{n_{j}} \text { for } j=1, \ldots, m
$$

where $G_{j}$ and $n_{j}$ are the respective transfer function and associated noise from the $j$-th imaging sensor. Assuming that no statistical information is available, find the image $f_{a}$ which yields a least squares fit between predicted and observed images, i.e., minimize

$$
\sum_{j=1}^{m}\left|\widehat{s_{j}}\left(\xi_{1}, \xi_{2}\right)-\widehat{f}_{a}\left(\xi_{1}, \xi_{2}\right) \widehat{G_{j}}\left(\xi_{1}, \xi_{2}\right)\right|^{2}
$$

After differentiating with respect to $\overline{\widehat{f}_{a}}$, the solution is found to be

$$
\widehat{f}_{a}\left(\xi_{1}, \xi_{2}\right)=\sum_{j=1}^{m} \widehat{s}_{j}\left(\xi_{1}, \xi_{2}\right) \widehat{\widetilde{G}}_{j}\left(\xi_{1}, \xi_{2}\right),
$$


where

$$
\widehat{\tilde{G}_{j}}\left(\xi_{1}, \xi_{2}\right)=\frac{\overline{\widehat{G_{j}}}\left(\xi_{1}, \xi_{2}\right)}{\sum_{k=1}^{m}\left|\widehat{G}_{k}\left(\xi_{1}, \xi_{2}\right)\right|^{2}},
$$

for $j=1, \ldots, m$. The synthesis filters $\left\{\tilde{G}_{j}\right\}_{j=1}^{m}$ are robust (and even optimal) with respect to any residual noise left from the decompositions that might remain after thresholding schemes have been utilized for denoising purposes. When filters of small finite support are desired, we use the method given in [11], which reduces the problem of finding the synthesis filters to solving a constrained matrix inversion problem. This method is particularly flexible as the support sizes of the synthesis filters can be controlled by a free parameter that balances between local and global conditioning.

Another solution to achieve perfect reconstruction is to slightly modify the analysis filters to be

$$
\widehat{G_{j}^{\prime}}\left(\xi_{1}, \xi_{2}\right)=\frac{\widehat{G_{j}}\left(\xi_{1}, \xi_{2}\right)}{\sqrt{\sum_{k=1}^{m}\left|\widehat{G}_{k}\left(\xi_{1}, \xi_{2}\right)\right|^{2}}},
$$

and to use

$$
\widehat{\tilde{G}}_{j}\left(\xi_{1}, \xi_{2}\right)=\frac{\overline{\widehat{G_{j}}}\left(\xi_{1}, \xi_{2}\right)}{\sqrt{\sum_{k=1}^{m}\left|\widehat{G}_{k}\left(\xi_{1}, \xi_{2}\right)\right|^{2}}},
$$

as the synthesis filters for $j=0, \ldots, m-1$. Note that this solution means that the implemented transform corresponds to a tight frame. However, we have found that for some constructions the MDP solutions perform better.

To emphasize the benefits of our proposed filter constructions, we show the differences in frequency responses for some representatives of the new shearlet filters and the non-subsampled contourlet transform (NSCT) filters in Figure 12. This illustration shows that whereas the NSCT filters may be constructed by using conventional filter design elements, their desired frequency responses do not truly match with 


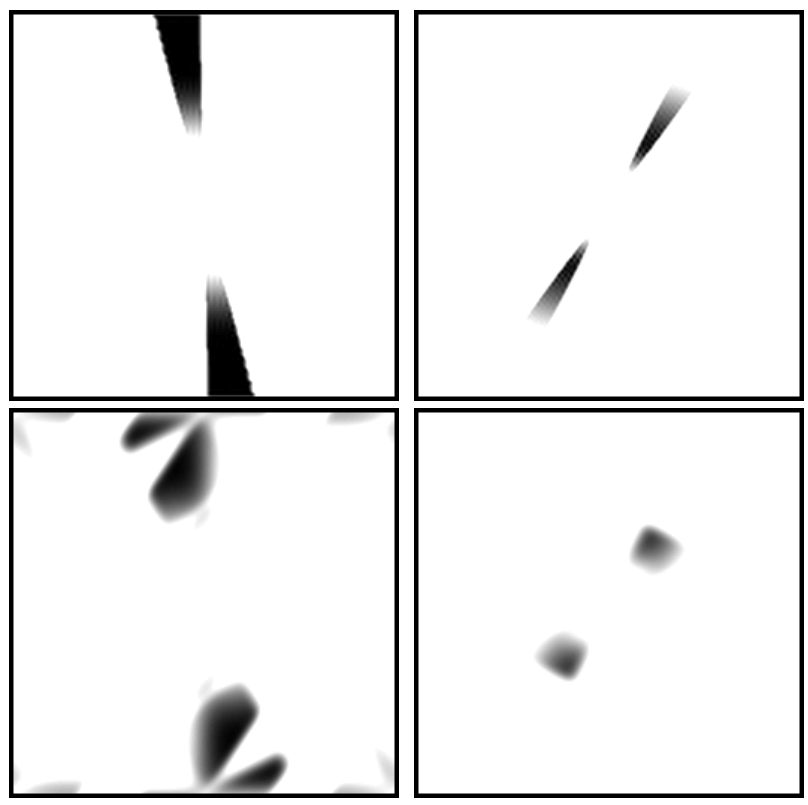

Figure 12. Comparison of filters design methods. The images on the top correspond to examples of the frequency responses of the newly constructed shearlet filters. The images on the bottom correspond to examples of the frequency responses of the NSCT for the same directional components.

the actual frequency responses. Nonetheless, the NSCT filters are very effective and have other advantages.

We can summarized the algorithm for the construction of the filters implementing the discrete wavelets with composite dilations as follows.

(1) Generate a sequence of points $\left\{\left(\xi_{1}^{n}, \xi_{2}^{n}\right)\right\} \in S$ filling densely the support region $S$ associated with the generator of the system of wavelets with composite dilations.

(2) Given $j$ and $\ell$, form filter $\widehat{G}_{j, \ell}$ by assigning the value at $\left(\bar{\eta}_{1}^{n}, \bar{\eta}_{2}^{n}\right)=\left\lceil\left(\xi_{1}^{n}, \xi_{2}^{n}\right) a^{j} b^{\ell}\right\rceil$ to be the average of the pre-image values of the generator function evaluated at $\left(\xi_{1}^{n}, \xi_{2}^{n}\right)$.

(3) Use synthesis filters $\widehat{\tilde{G}}_{j, \ell}\left(\xi_{1}, \xi_{2}\right)=\frac{\overline{\widehat{G}}_{j, \ell}\left(\xi_{1}, \xi_{2}\right)}{\sum_{j, \ell}\left|\widehat{G}_{j, \ell}\left(\xi_{1}, \xi_{2}\right)\right|^{2}}$, solve for synthesis filters by solving the matrix system as described in [11], or use (4) and (5).

The filterbank implementation is highly efficient as it requires $O\left(N^{2} \log N\right)$ operations for an $N \times N$ image. 


\section{Numerical Experiments}

In this section, we present several numerical experiments on image restoration and enhancement to demonstrate the effectiveness of the wavelets with composite dilations and their discrete implementation. The simulations were done using Matlab on a Windows system with Intel Pentium M/1.73 GHz processor.

4.1. Denoising. In the first set of experiments, we illustrate the denoising capability of the new wavelets with composite dilations by means of hard thresholding.

The objective of this problem is to recover an image $x$, given noisy observations

$$
y=x+\gamma,
$$

where $\gamma$ is zero-mean white Gaussian noise with variance $\sigma^{2}$. By adapting the standard wavelet shrinkage approach [37], we apply a hard threshold on the subband coefficients of several versions of composite wavelets decompositions. In particular, we choose the threshold $T_{j}=K \sigma_{j}$, where $\sigma_{j}^{2}$ is the noise variance in each subband and $K$ is a constant. In our experiments, we set $K=2$ for all subbands.

To assess the denoising performance of our method, we compare it against three different competing discrete multiscale transforms: the nonsubsampled wavelet transform (NSWT), the second generation curvelet transform (curv) [7], and the nonsubsampled contourlet transform (NSCT). The discrete wavelets with composite dilations we have tested are the new shearlet transform ( $a b$-shear), the conebased hyperbolic transform (c-hyper), the hyperbolic transform (hyper), the starlike transform given in Construction 1 (ab-star). Specifically, the new constructions are done using the pseudo-code shown in the appendix so that the frequency plane decompositions correspond to the decompositions displayed in Figures 2 (left side), 3,6 , and 8 . The value of $N_{0}$ is set to the size of the image the transform is being applied to. For the sake of comparison, we have also included the original implementation of the shearlet transform (shear) [17]. The peak signal-to-noise ratio 
(PSNR) is used to measure the performance of the different transforms. Given an $N \times N$ image $x$ and its estimate $\tilde{x}$, the PSNR in decibels $(\mathrm{dB})$ is defined as

$$
P S N R=20 \log _{10} \frac{255 N}{\|x-\tilde{x}\|_{F}}
$$

where $\|\cdot\|_{F}$ is the Frobenius norm. In Tables I and II, we show the results obtained using various discrete transforms on the Peppers and Barbara images, respectively. The highest PSNR for each experiment is shown in bold. As it can be seen from the tables, all our new transforms provide superior or comparable results to that obtained using NSWT, NSCT and curvelets. Indeed, in some cases, the composite wavelet transforms provide improvement of nearly $1 \mathrm{~dB}$ or more compared to the competing algorithms. Figures 13, 14, 15 and 16 show some of the reconstructed images for these various experiments.

Table I: Denoising results using Peppers image.

\begin{tabular}{|c||c|c|c|c|c|c|c|c|c|}
\hline$\sigma$ & Noisy & ab-shear & c-hyper & hyper & ab-star & NSWT & shear & NSCT & curv \\
\hline \hline 10 & 28.17 & $\mathbf{3 4 . 2 8}$ & 33.70 & 33.91 & 33.45 & 33.71 & 34.05 & 33.81 & 32.36 \\
\hline 20 & 22.15 & $\mathbf{3 1 . 8 2}$ & 31.01 & 31.28 & 30.83 & 31.19 & 31.78 & 31.60 & 29.65 \\
\hline 30 & 18.63 & $\mathbf{3 0 . 2 5}$ & 29.35 & 29.72 & 29.26 & 29.43 & 30.13 & 30.07 & 28.25 \\
\hline 40 & 16.13 & $\mathbf{2 9 . 0 1}$ & 28.12 & 28.40 & 28.04 & 28.09 & 28.86 & 28.85 & 27.28 \\
\hline 50 & 14.20 & $\mathbf{2 7 . 9 8}$ & 27.17 & 27.33 & 26.99 & 27.04 & 27.90 & 27.82 & 26.46 \\
\hline
\end{tabular}

Table II: Denoising results using Barbara image.

\begin{tabular}{|c||c|c|c|c|c|c|c|c|c|}
\hline$\sigma$ & Noisy & ab-shear & c-hyper & hyper & ab-star & NSWT & shear & NSCT & curv \\
\hline \hline 10 & 28.17 & 33.47 & $\mathbf{3 3 . 9 7}$ & 33.81 & 32.29 & 31.58 & 33.12 & 33.01 & 29.16 \\
\hline 20 & 22.15 & 30.38 & 30.41 & $\mathbf{3 0 . 4 9}$ & 29.02 & 27.23 & 30.07 & 29.41 & 25.46 \\
\hline 30 & 18.63 & 28.44 & 28.37 & $\mathbf{2 8 . 5 3}$ & 27.02 & 25.10 & 28.16 & 27.24 & 24.42 \\
\hline 40 & 16.14 & $\mathbf{2 6 . 9 3}$ & 26.86 & 26.75 & 25.55 & 24.02 & 26.59 & 25.79 & 23.81 \\
\hline 50 & 14.20 & 25.59 & $\mathbf{2 5 . 7 8}$ & 25.35 & 24.06 & 23.37 & 25.39 & 24.79 & 23.33 \\
\hline
\end{tabular}

In Table III, we report average runtime of the different transforms in filtering noisy images. Note that NSWT and curv use preconstructed filters. As a result they are very fast and run in less than a minute. The performance of our composite wavelet transforms can be significantly enhanced by implementing them in a parallel form and using other modifications, but this is beyond the scope of this paper. 


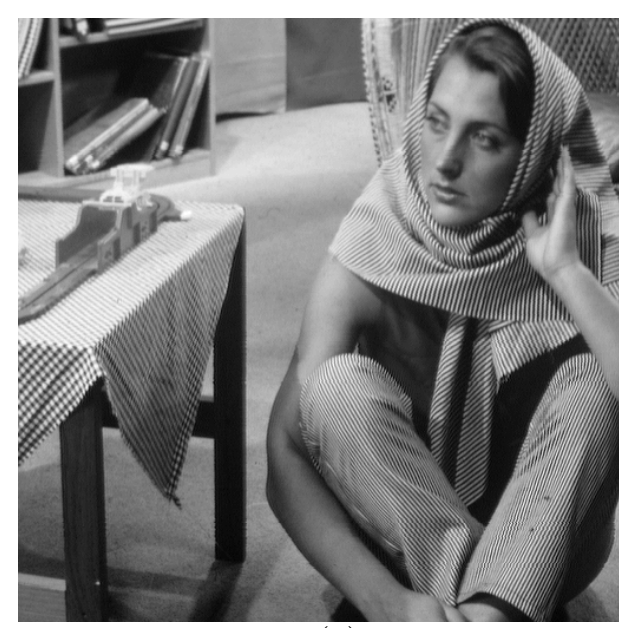

(a)

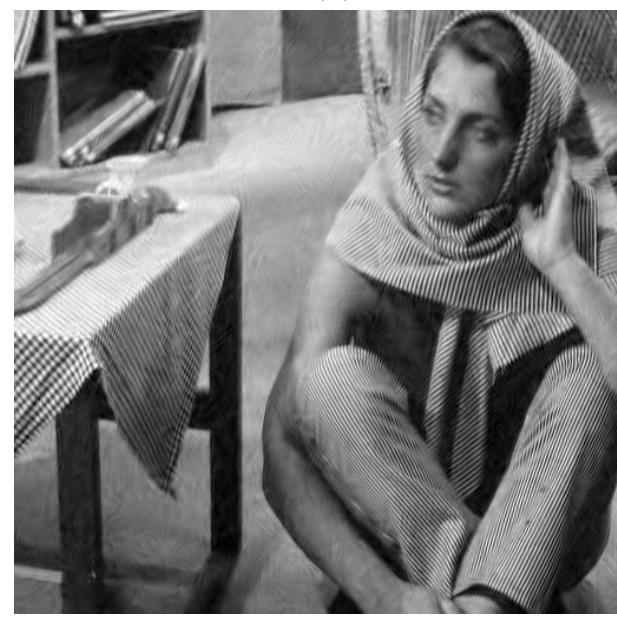

(c)

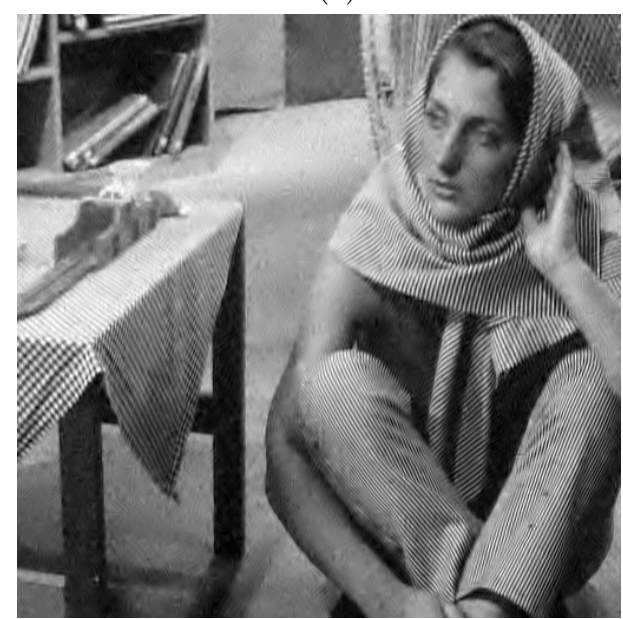

(e)

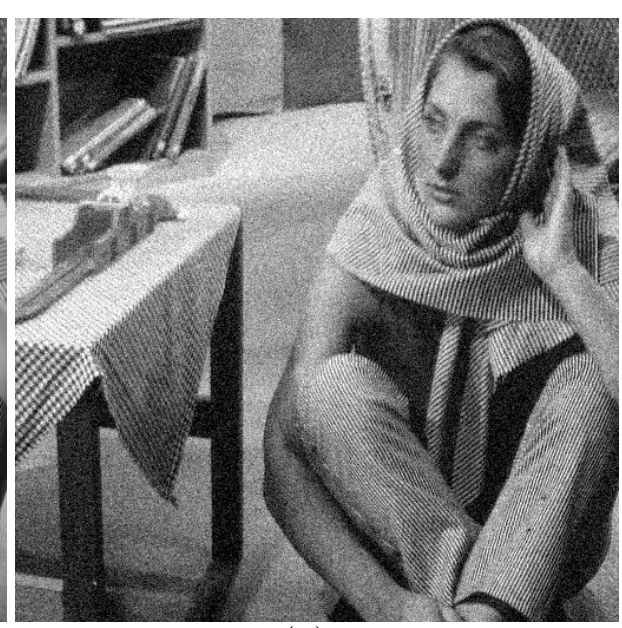

(b)

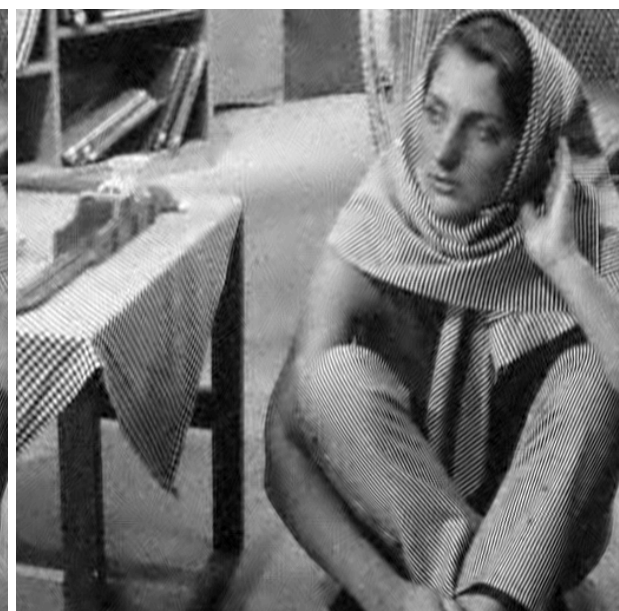

(d)

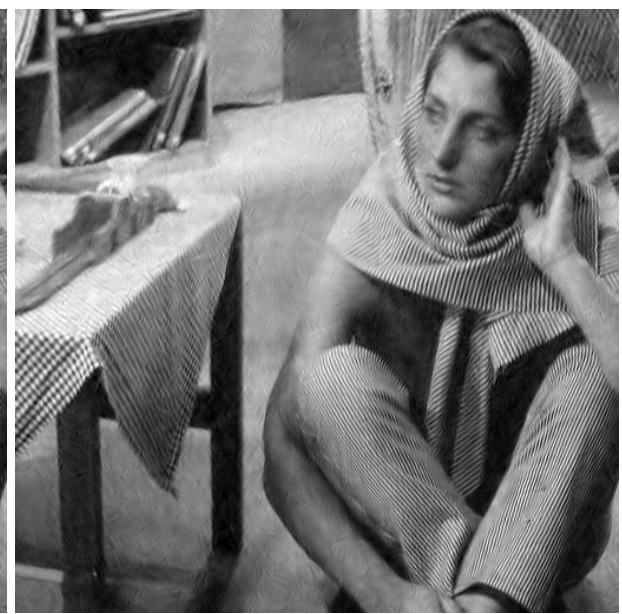

(f)

Figure 13. Denoising experiments with a Barbara image. (a) Original image. (b) Noisy image with $\sigma=20, \mathrm{PSNR}=22.15 \mathrm{~dB}$. (c) Restored image using ab-shear, PSNR $=30.38 \mathrm{~dB}$. (d) Restored image using c-hyper, PSNR $=30.41 \mathrm{~dB}$. (e) Restored image using hyper, PSNR=30.49 dB. (f) Restored image using ab-star, $\mathrm{PSNR}=29.02 \mathrm{~dB}$. 




(a)

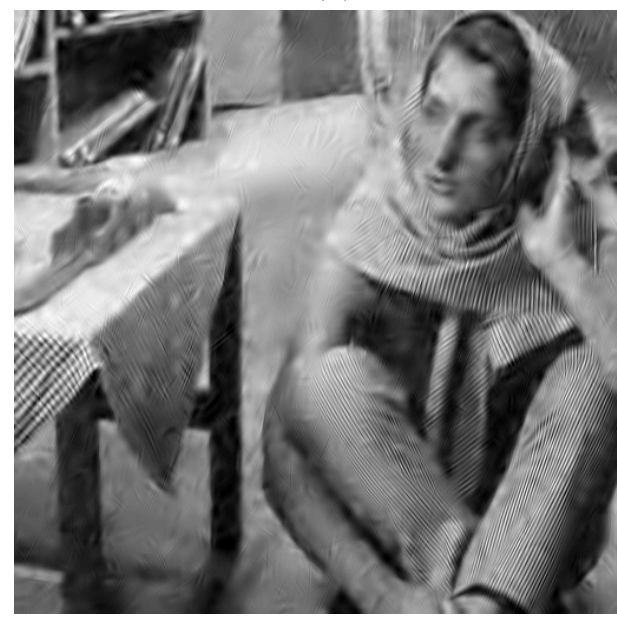

(c)

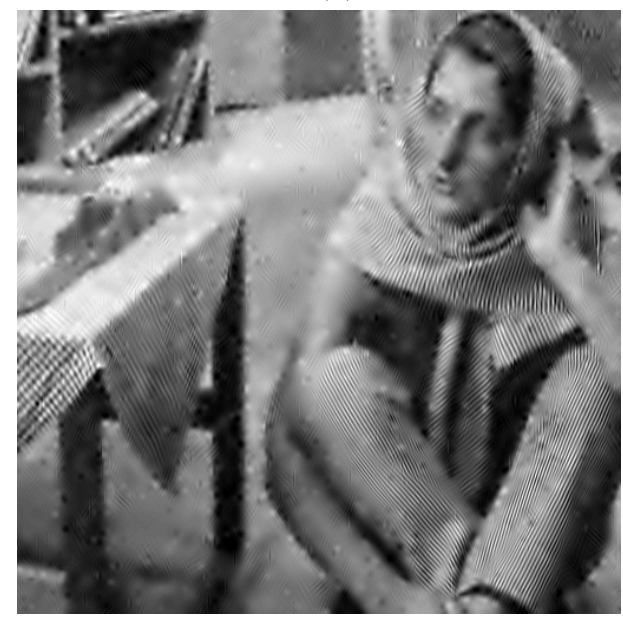

(e)

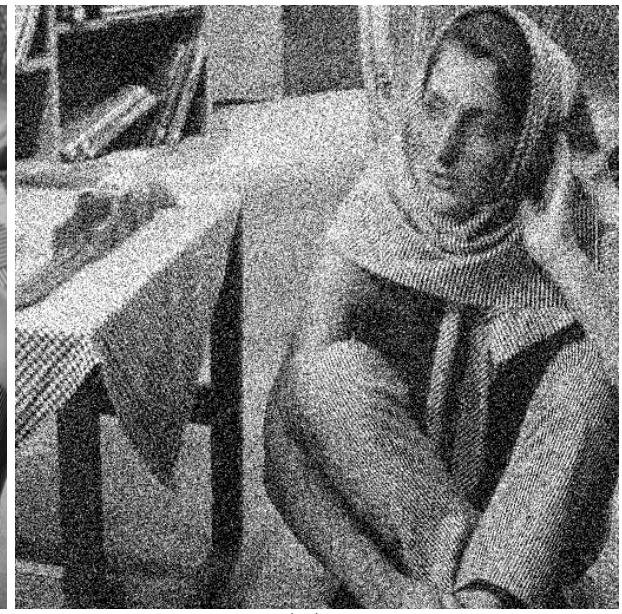

(b)



(d)



(f)

Figure 14. Denoising experiments with a Barbara image. (a) Original image. (b) Noisy image with $\sigma=50, \mathrm{PSNR}=14.20 \mathrm{~dB}$. (c) Restored image using ab-shear, PSNR=25.59 dB . (d) Restored image using c-hyper, PSNR $=25.78 \mathrm{~dB}$. (e) Restored image using hyper, PSNR=25.35 dB. (f) Restored image using ab-star, $\mathrm{PSNR}=24.06 \mathrm{~dB}$. 


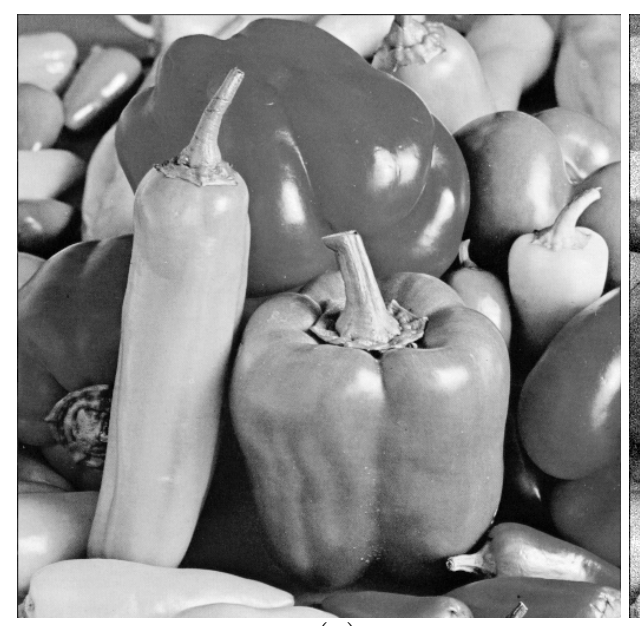

(a)

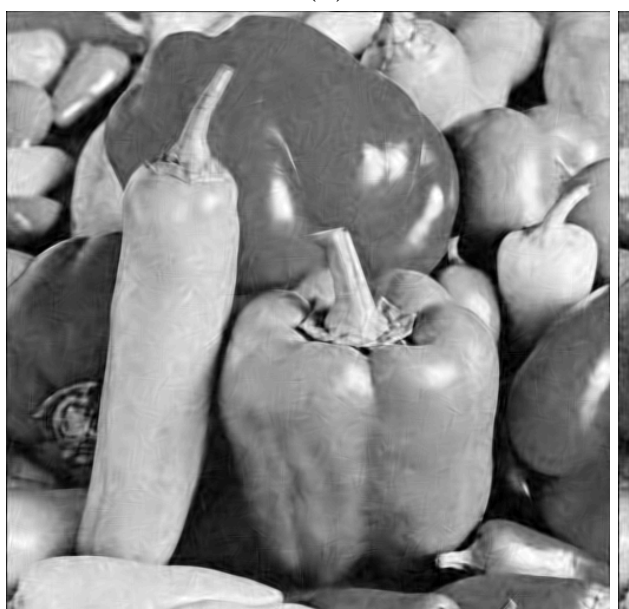

(c)

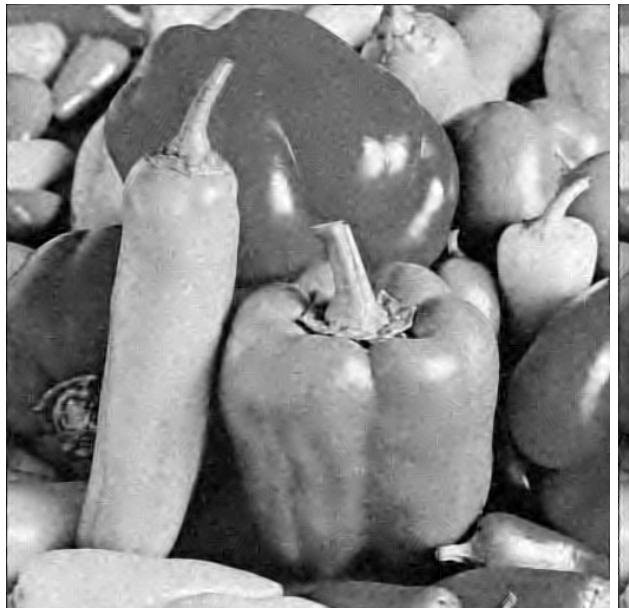

(e)



(b)

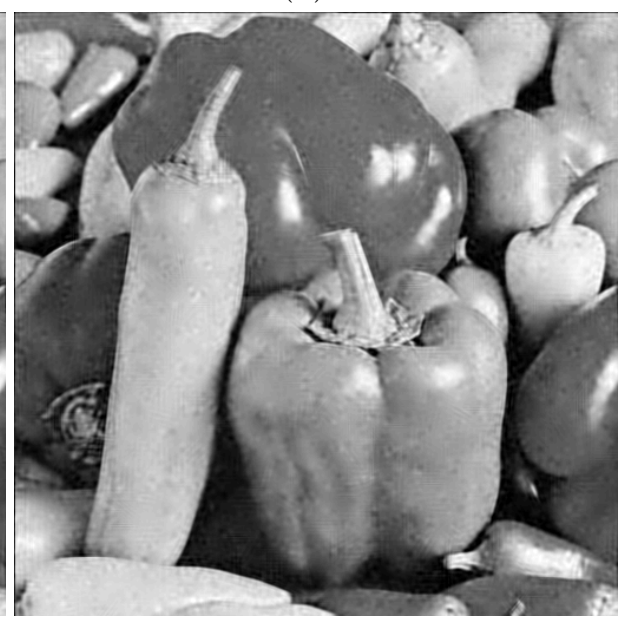

(d)

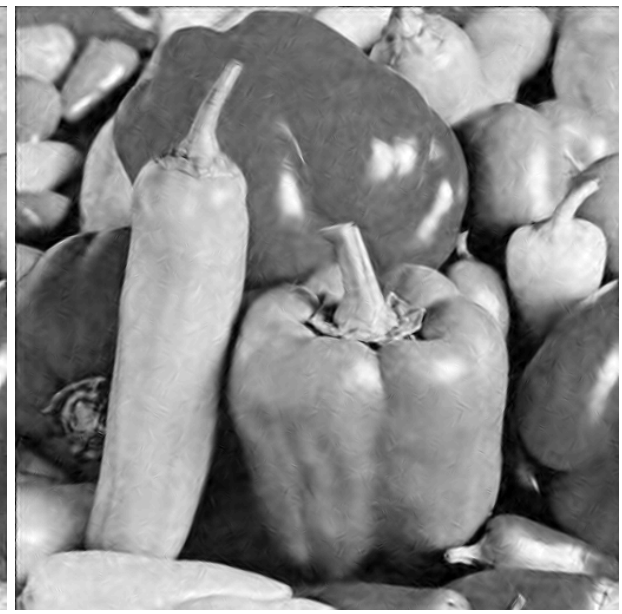

(f)

Figure 15. Denoising experiments with a Peppers image. (a) Original image. (b) Noisy image with $\sigma=20, \mathrm{PSNR}=22.15 \mathrm{~dB}$. (c) Restored image using ab-shear, PSNR=31.82 dB . (d) Restored image using c-hyper, PSNR=31.01 dB. (e) Restored image using hyper, PSNR $=31.28 \mathrm{~dB}$. (f) Restored image using ab-star, $\mathrm{PSNR}=30.83 \mathrm{~dB}$. 


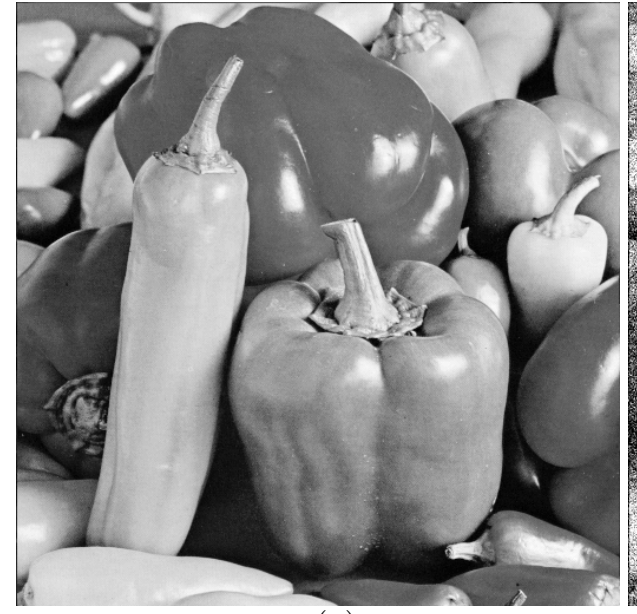

(a)

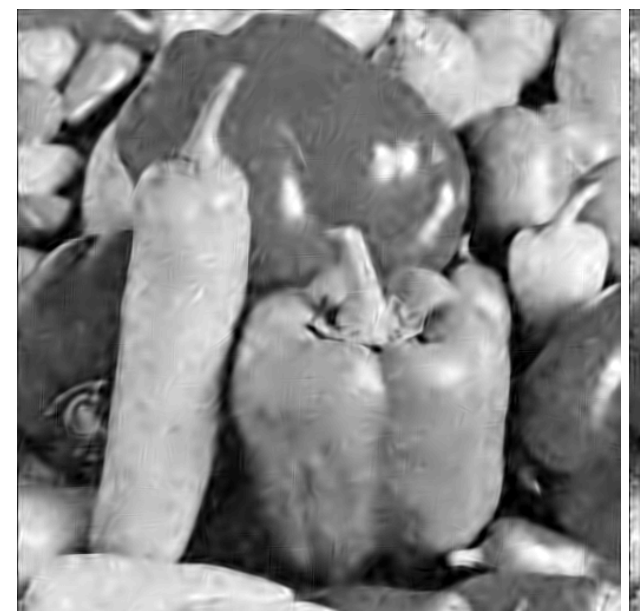

(c)

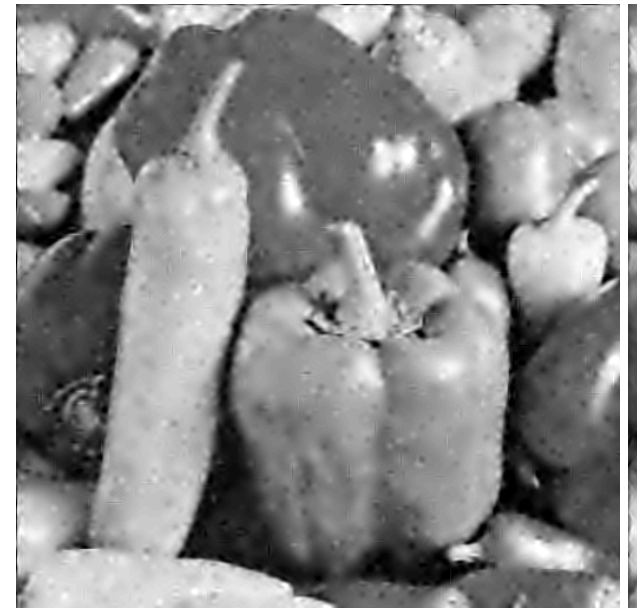

(e)

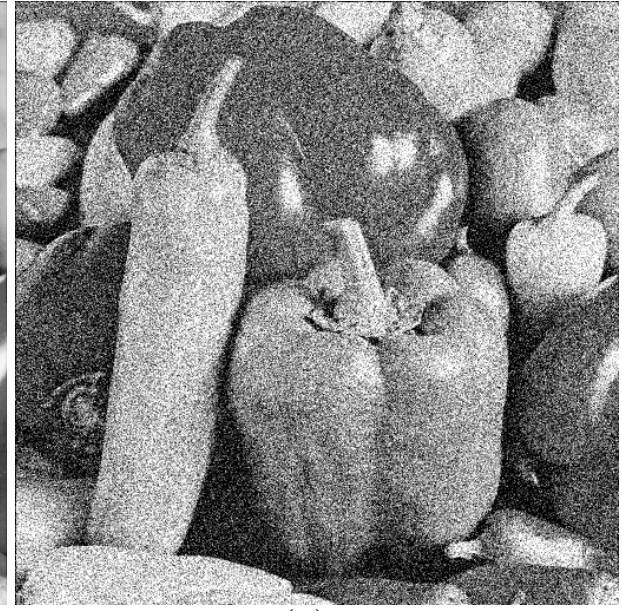

(b)

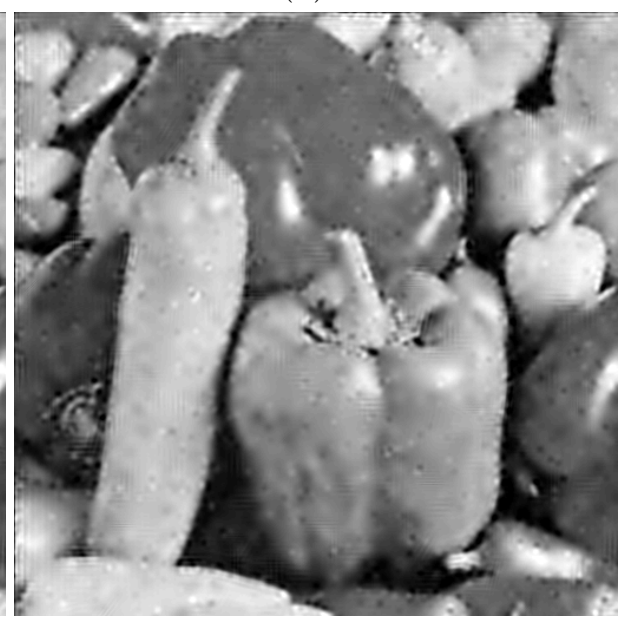

(d)

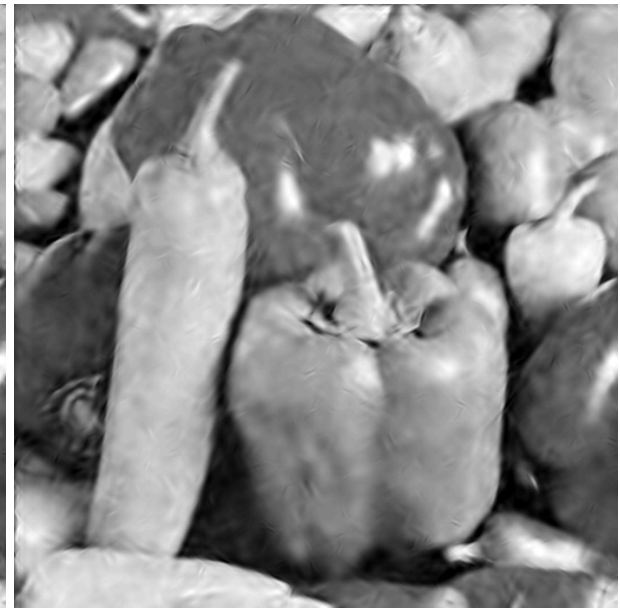

(f)

Figure 16. Denoising experiments with a Peppers image. (a) Original image. (b) Noisy image with $\sigma=50, \mathrm{PSNR}=14.20 \mathrm{~dB}$. (c) Restored image using ab-shear, PSNR=27.98 dB . (d) Restored image using c-hyper, PSNR=27.17 dB. (e) Restored image using hyper, PSNR=27.33 dB. (f) Restored image using ab-star, $\mathrm{PSNR}=26.99 \mathrm{~dB}$. 
Table III: Average runtime in seconds.

\begin{tabular}{|c|c|c|c|c|c|c|c|c|}
\hline Image Size & ab-shear & c-hyper & hyper & ab-star & NSWT & shear & NSCT & curv \\
\hline \hline $256 \times 256$ & 63.27 & 113.64 & 77.53 & 13.23 & 0.58 & 35.93 & 45.89 & 6.86 \\
\hline $512 \times 512$ & 198.39 & 306.56 & 233.60 & 42.03 & 3.02 & 115.56 & 286.90 & 32.21 \\
\hline
\end{tabular}

4.2. Enhancement. The objective of image enhancement is to improve the visual or perceptual quality of an image in a certain sense. For example, a simple and well-known method to visually improve images with poor intensity is contrast enhancement using histogram equalization.

Since edges frequently contain the dominant information of an image, an effective way to improve the contrast of the image consists in enhancing the edges. This approach has been widely used in astronomical and medical imaging, especially using methods based on multiscale image decomposition. However, in most of these methods, the image is decomposed in a separable fashion, without taking full advantage of the geometric information associated with the edges [35, 30]. By contrast, a multiscale decomposition which is able to take advantage of directional features, such as curvelets, shearlets or wavelets with composite dilations, is much more effective in dealing with the edges and other directional information [16, 47, 43, 12]. At the same time, it is well-known that natural images have their energy strongly concentrated at low frequencies. Since directional information is related mostly to mid- and high-frequency components, the lowpass information can hinder our ability to efficiently capture and analyze these features. Therefore, scale information needs to be used in conjunction with directional information in applications where it is important to distinguish features of different sizes. To this end, in this section, we exploit the directional and multiscale properties of the newly designed wavelets with composite dilations for a new algorithm for image enhancement.

Our approach is based on a mapping function which amplifies weak edges and suppresses noise. Specifically, we introduce a new adaptive nonlinear mapping function that incorporates the nonnegative garrote shrinkage functions. This approach provides a good compromise between hard and soft shrinkage rules and allows one 
to avoid amplifying noise and remove small noise perturbations. Our mapping $f$ is defined as

$$
f(y)=\left\{\begin{array}{l}
0 \quad \text { if }|y|<T_{1}, \\
\operatorname{sign}(y) T_{2}+\bar{\alpha}\left(\operatorname{sigm}\left(c\left(g_{y}-\beta\right)\right)-\operatorname{sigm}\left(-c\left(g_{y}+\beta\right)\right)\right) \text { if } T_{2} \leq|y| \leq T_{3} \\
y \quad \text { otherwise. }
\end{array}\right.
$$

where $\operatorname{sigm}(y)=\left(1+e^{-y}\right)^{-1}, y \in[-1,1], \bar{\alpha}=\alpha\left(T_{3}-T_{2}\right), \beta \in(0,1), c$ is a gain factor, $0 \leq T_{1} \leq T_{2}<T_{3} \leq 1$, and $g_{y}=\frac{\text { garrote }_{T_{2}}(y)}{T_{3}-T_{2}}$, with

$$
\operatorname{garrote}_{T_{2}}(y)=\left\{\begin{array}{lll}
0, & \text { for } & |y| \leq T_{2} \\
y-\frac{T_{2}^{2}}{y}, & \text { for } & |y|>T_{2}
\end{array}\right.
$$

Notice that $\alpha$ can be computed by $\alpha=(\operatorname{sigm}(c(1-\beta))-\operatorname{sigm}(-c(1+\beta)))^{-1}$. Here, $\beta$ and $c$ determine the threshold and the rate of enhancement, respectively. As shown in Figure 17, when the mapping $f$ is applied to the composite wavelet transform of an image, only the values falling in the interval $\left[T_{2}, T_{3}\right]$ are modified for enhancement, while the values in $\left[0, T_{1}\right]$ are suppressed. These parameters can be adaptively estimated by using the robust median operator [9] and the noise variance in each subband of the image decomposition [44]. For example, $T_{1}, T_{2}$, and $T_{3}$ for the subband $j$ can be chosen as $p \sigma_{j}, q \sigma_{j}, r \sigma_{j}$, respectively, where $\sigma^{2}$ is the noise variance of the input image and $\sigma_{j}^{2}$ is the noise variance of the $j$ th subband and $p, q$ and $r$ are user defined values. Through this nonlinear function, the values of the transformed image in each subband are pointwise modified by

$$
\tilde{y_{k}}=y_{k_{\max }} f\left(\frac{y_{k}}{y_{k_{\max }}}\right)
$$

where $1 \leq k \leq m, y_{k}$ is the output of the $k$ th channel of the filter bank, and $y_{k_{\max }}$ is the maximum absolute amplitude of $y_{k}$.

To summarize, our image enhancement algorithm based on the discrete wavelet transform with composite dilations consists of the following steps: 
(1) Estimate the noise standard deviation in the $N \times N$ input image using the robust median operator [9].

(2) Pass the input image through the analysis part of the filter bank obtaining a set of $m$ subbands, each corresponding to a given scale and direction. Each subband contains $N^{2}$ coefficients.

(3) For each subband:

(a) Calculate the noise standard deviation [43].

(b) Use the nonlinear mapping function to modify the subband coefficients.

(4) Pass the modified coefficients through the synthesis part of the filter bank and reconstruct the enhanced image.

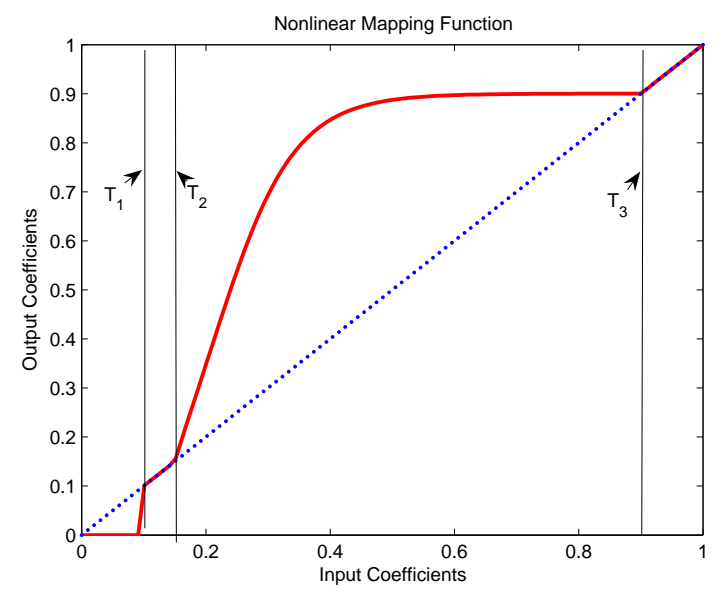

Figure 17. Enhancement map: $\beta=0.20, c=25, T_{1}=0.1, T_{2}=$ $0.15, T_{3}=0.9$.

Even though there is no standard way of evaluating the quality of enhanced images, a number of metrics have been proposed to quantitative analyze the enhanced images, such as the Universal Quality Index (UQI) [46]. UQI models image distortion as a combination of three factors: loss of correlation, luminance distortion and contrast distortion. UQI takes values between -1 and 1 , with 1 providing the best estimate of quality. 


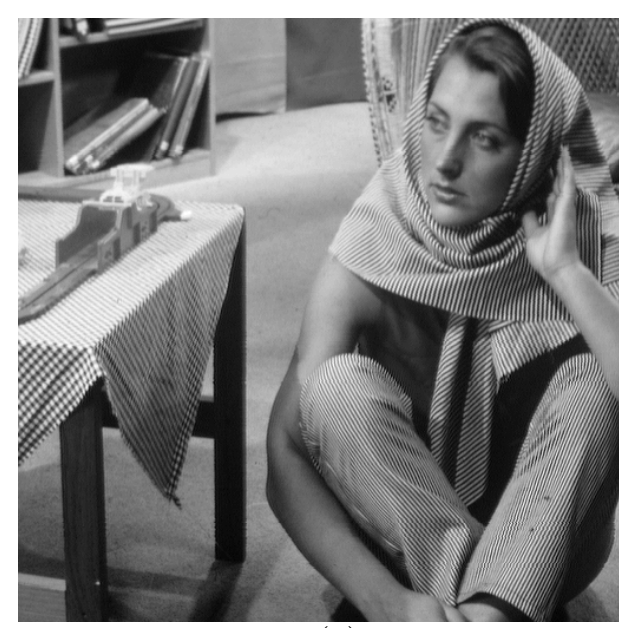

(a)



(c)

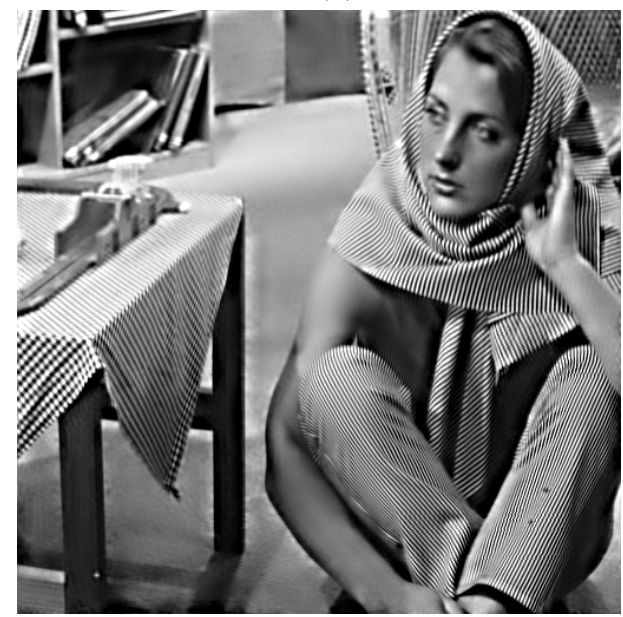

(e)

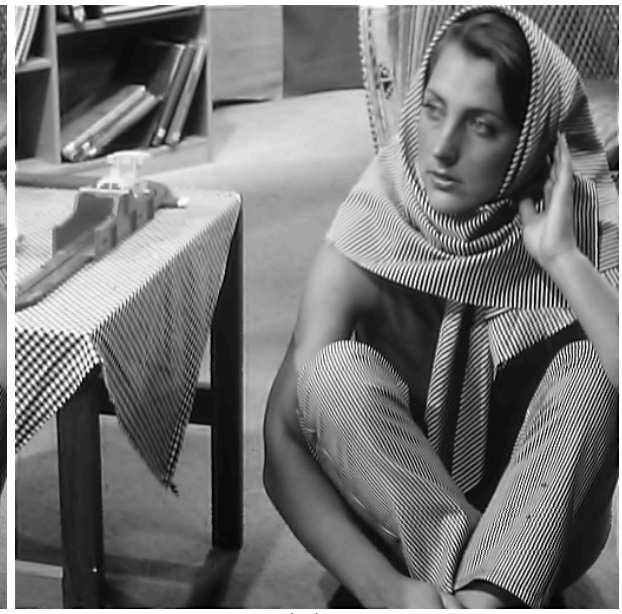

(b)

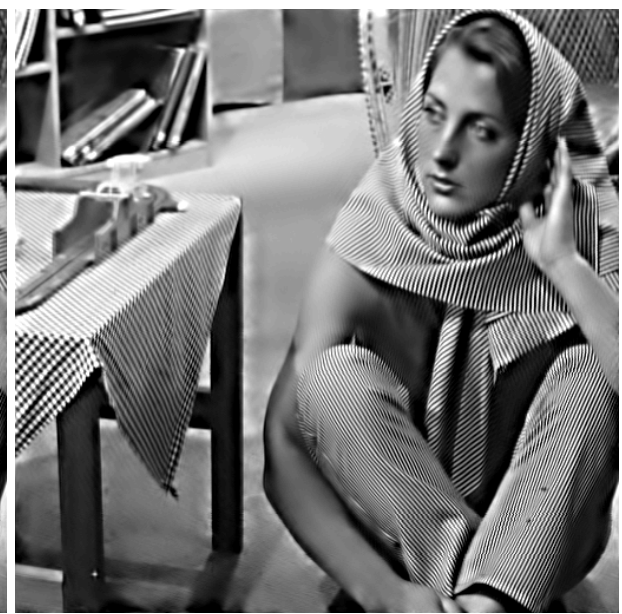

(d)

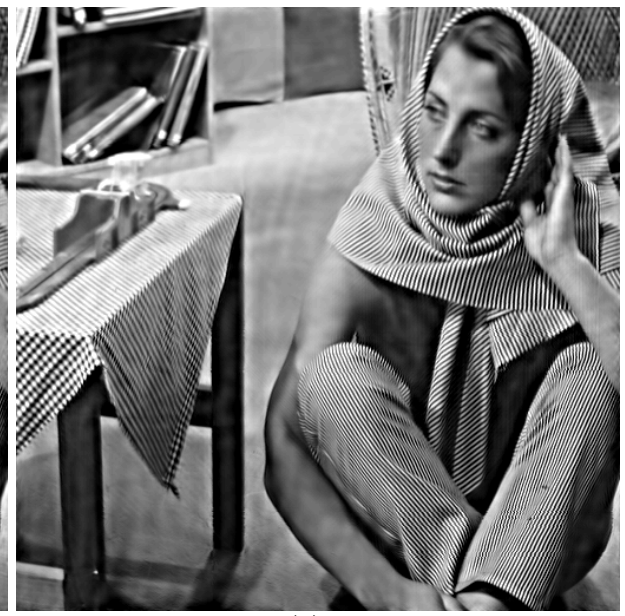

(f)

Figure 18. Enhancement experiments with a Barbara image. (a) Original image. (b) Enhanced using NSWT. (c) Enhanced using ab-shear. (d) Enhanced using hyper. (e) Enhanced using c-hyper. (f) Enhanced using ab-star. 


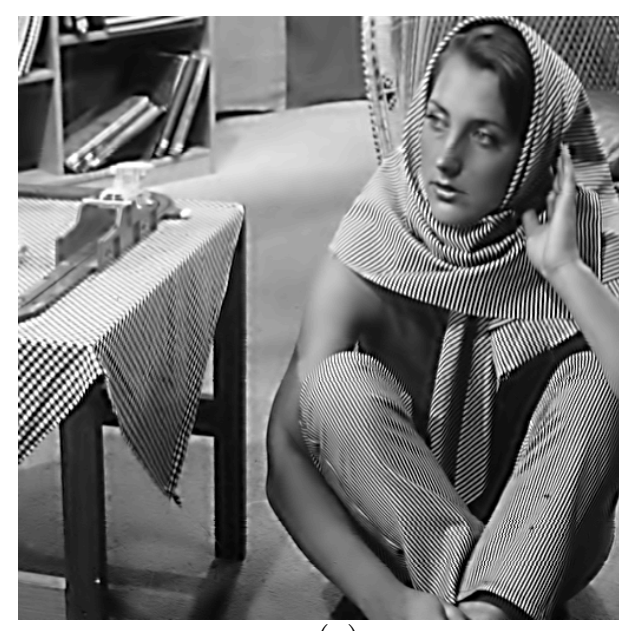

(a)

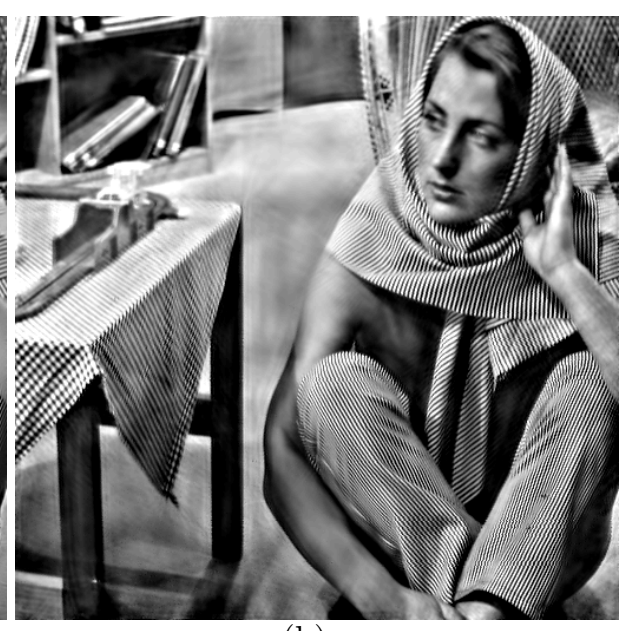

(b)

FiguRE 19. Enhancement experiments with a Barbara image. (a) Enhanced using NSCT. (b) Enhanced using curv.

We have compared our enhancement method against the NSWT, NSCT and curv using the same nonlinear map for all our tests. In the first set of experiments, we used the classical Barbara image and the following parameters $\beta=0.22$ and $c=15$. Figures 18(b)-(f) and Figures 19(a)-(b) indicate that our proposed enhancement algorithm works better using the wavelets with composite dilations developed in this paper than using the NSWT and curv. The ab-shearlet tiling-based enhancement gives results visually similar to that of NSCT.

In the second set of experiments, the input image is a Zebra image, as shown in Figure 20(a). For this set of experiments, we chose $\beta=0.20$ and $c=0.15$ for this set of experiments. Figures 20(b)-(f) and Figures 21(a)-(b), show the results obtained by various transforms. As we see consistently from all these figures, the enhancement algorithm based on wavelets with composite dilations is very effective in enhancing the weak edges and retaining textures while suppressing the noise. This can be confirmed by looking at the UQI metrics in Table III.

4.3. Deconvolution Example. When the model of the degradation of an image is described as a convolution operation, the processing of recovering the original 


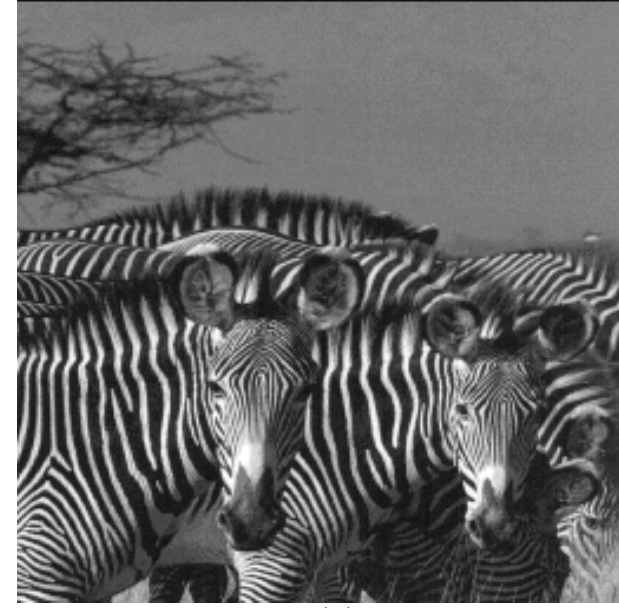

(a)



(c)

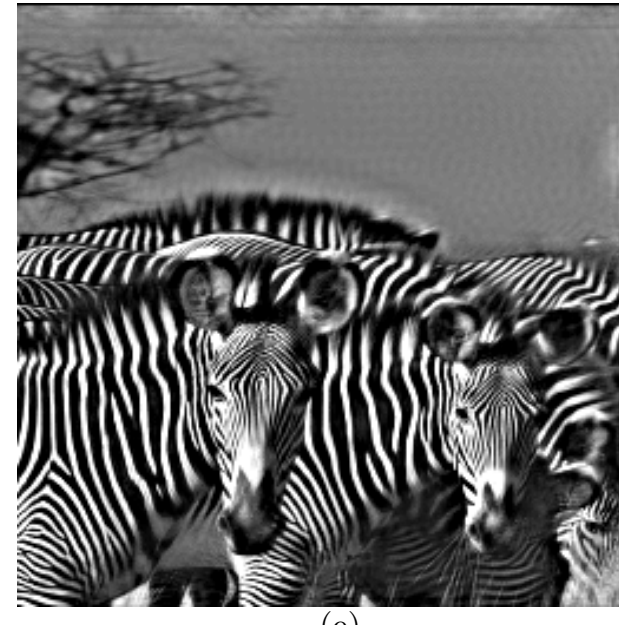

(e)

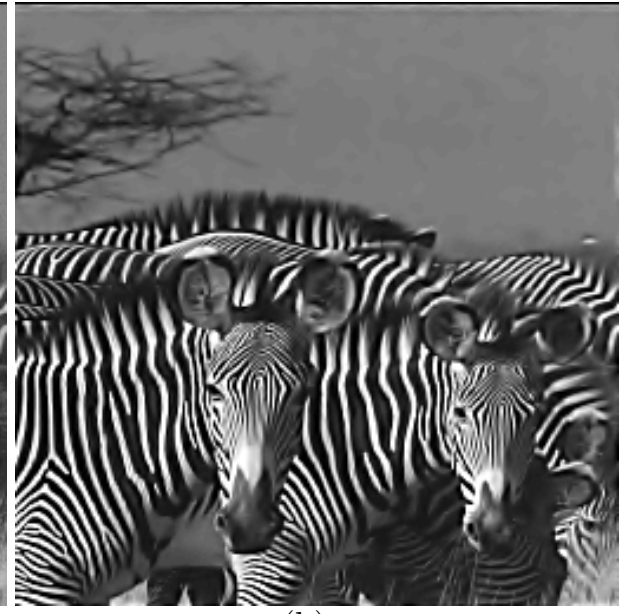

(b)

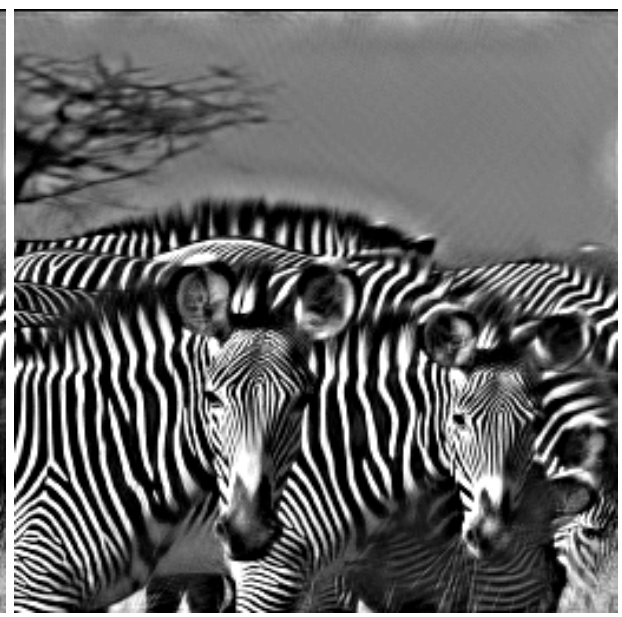

(d)

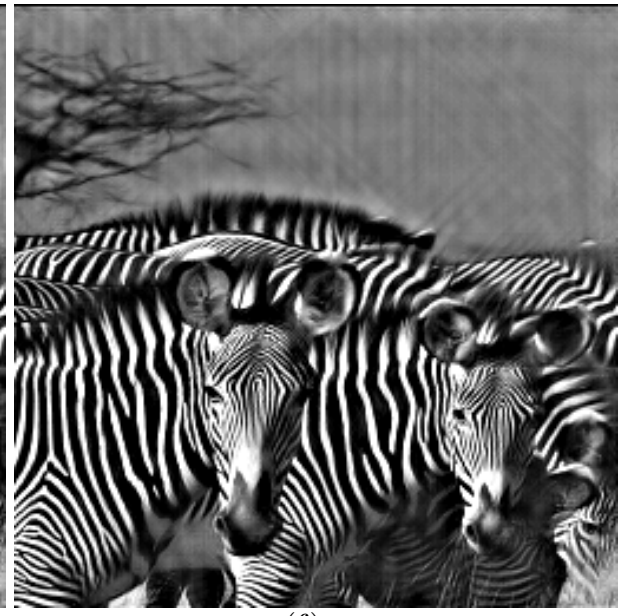

(f)

Figure 20. Enhancement experiments with a Zebra image. (a) Original image. (b) Enhanced using NSWT. (c) Enhanced using ab-shear. (d) Enhanced using hyper. (e) Enhanced using c-hyper. (f) Enhanced using ab-star. 


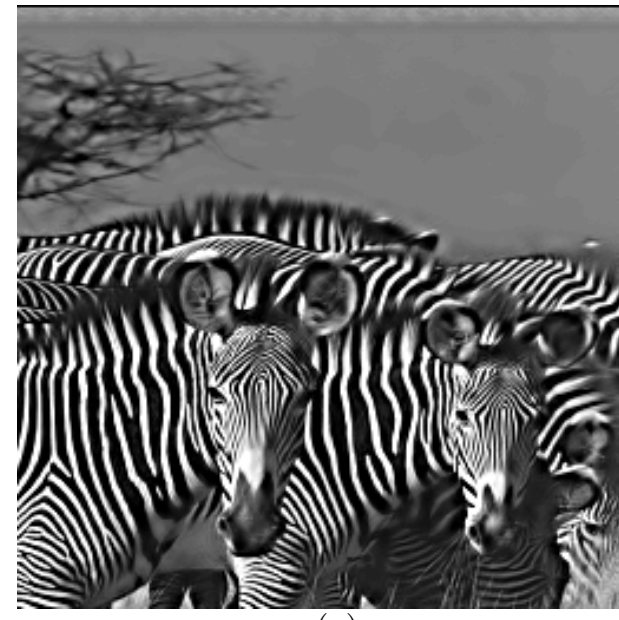

(a)

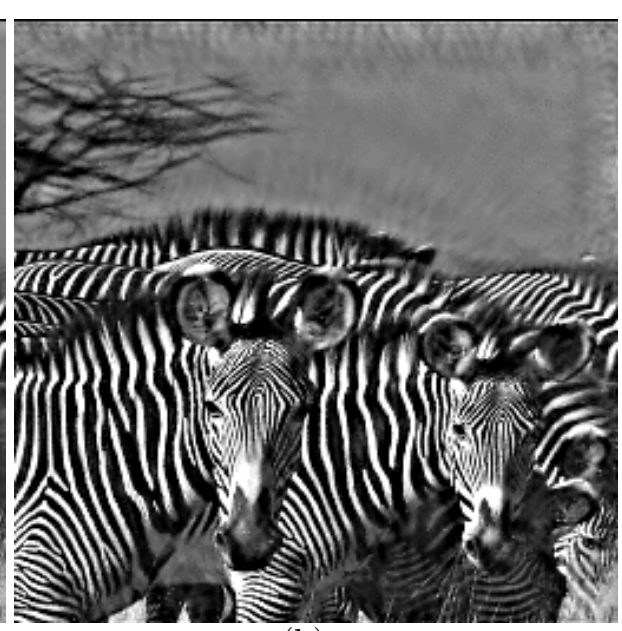

(b)

FIGURE 21. Enhancement experiments with a Zebra image. (a) Enhanced using NSCT. (b) Enhanced using curv.

Table III: UQI results for image enhancement experiments.

\begin{tabular}{|c||c|c|c|c|c|c|c|}
\hline Image & ab-shear & c-hyper & hyper & ab-star & NSWT & NSCT & curv \\
\hline Barbara & 0.6918 & $\mathbf{0 . 7 0 2 7}$ & 0.6953 & 0.6672 & 0.6750 & 0.6923 & 0.6687 \\
\hline Zebra & 0.6538 & 0.6853 & $\mathbf{0 . 6 8 7 3}$ & 0.6827 & 0.6800 & 0.6827 & 0.6820 \\
\hline
\end{tabular}

image from the degraded blurred image is commonly referred to as deconvolution. This inverse processes is known to be an ill-posed problem.

In this subsection, we give some preliminary experiments that indicate the ability of using a hyperbolic composite wavelet to better regularize the deconvolution process. The key point to make is that regularization for this type of problem is best done by using a representation that can sparsely represent the image and the convolution operator.

Let $\gamma$ denote an $N \times N$ array of samples from a zero mean additive white Gaussian noise $(\mathrm{AWGN})$ with variance $\sigma^{2}$. In addition, we let the $N \times N$ arrays $y$ and $x$ denote the observed image and the image to be estimated, respectively. When the blurring function is symmetric about the origin and a reflexive boundary is assumed for the finite discretization of the problem, the convolution is diagonalizable in the 
DCT domain. Thus we write the deconvolution problem in the DCT domain as

$$
Y\left(k_{1}, k_{2}\right)=\mathcal{H}\left(k_{1}, k_{2}\right) X\left(k_{1}, k_{2}\right)+\Gamma\left(k_{1}, k_{2}\right)
$$

where $Y\left(k_{1}, k_{2}\right), \mathcal{H}\left(k_{1}, k_{2}\right), X\left(k_{1}, k_{2}\right)$ and $\Gamma\left(k_{1}, k_{2}\right)$ are the 2D DCTs of $y, h, x$, and $\gamma$, respectively, for $1 \leq k_{1}, k_{2} \leq N$. Figure 22 displays a contour plot of a typical out-of-focus blur in the DCT domain and also shows the corresponding hyperbolic composite decomposition when we consider the transform that results when the convolution is implemented with a reflexive boundary.

Using the regularized inverse operator $H_{\alpha}\left(k_{1}, k_{2}\right)=\mathcal{H}\left(k_{1}, k_{2}\right) /\left(\mathcal{H}\left(k_{1}, k_{2}\right)^{2}+\alpha\right)$ for some regularizing parameter $\alpha \in R^{+}$, an image estimate in the DCT domain is given by

$$
X_{\alpha}\left(k_{1}, k_{2}\right)=H_{\alpha}\left(k_{1}, k_{2}\right) Y\left(k_{1}, k_{2}\right),
$$

for $1 \leq k_{1}, k_{2} \leq N$. This type of regularization applied is often referred to as Tikhonov-regularization. Let $G_{j, \ell}$ denote the DCT of the composite wavelet filters $g_{j, \ell}$ for a given choice of $j$ and $\ell$. The coefficients of an estimate of the image for a given regularization parameter $\alpha_{j, \ell}$ can be computed in the DCT domain as

$$
X_{\alpha_{j, \ell}}\left(k_{1}, k_{2}\right)=H_{\alpha_{j, \ell}}\left(k_{1}, k_{2}\right) G_{j, \ell}\left(k_{1}, k_{2}\right) Y\left(k_{1}, k_{2}\right)
$$

for $1 \leq k_{1}, k_{2} \leq N$. Taking advantage of the hyperbolic-based decomposition, we can adaptively control the regularization parameter to be the best suited for each hyperbolic supported region. The final estimate is then found by inverting the composite wavelet transform using these estimated coefficients.

We compared this method against the standard Tikhonov-regularization using the Barbara image and out-of-focus point spread function shown in Figure 22. The results are provided in Table IV for various levels of noise given in terms of the socalled blurred signal-to-noise-ratio (BSNR) [11]. This is a commonly used metric for measuring the severity of blur and noise, which, for an image of size $N \times N$, is 
defined by

$$
B S N R=10 \log _{10}\left(\frac{\|(x * h)-\mu(x * h)\|_{2}^{2}}{N^{2} \sigma^{2}}\right),
$$

where $\mu(x * h)$ denotes the mean of $x * h$, and is measured in decibels.


FIGURE 22. The image on the left is the out-of-focus point spread function displayed in the DCT domain. The image on the right is the decomposition based on the hyperbolic composite wavelet when implemented by convolution with a reflexive boundary.

Table IV: Deconvolution results using the Barbara image.

\begin{tabular}{|c||c|c|c|c|}
\hline$B S N R$ & 30 & 35 & 40 & 45 \\
\hline hyper & $\mathbf{2 5 . 9 0}$ & $\mathbf{2 7 . 4 1}$ & $\mathbf{2 9 . 0 9}$ & $\mathbf{3 1 . 1 2}$ \\
\hline Tik & 24.86 & 26.04 & 27.76 & 29.73 \\
\hline
\end{tabular}

Our experiments indicate the potential of hyperbolic-based decompositions schemes for the problem of deconvolution. It is clear that there are other blurring functions that exhibit patterns containing valleys (low magnitude values) that follow a hyperbolic distribution. In these cases, an improved regularization is possible when the image representation is sufficiently sparse and it enables a control of the regularization parameters by isolating the locations of the valleys.

\section{Conclusion}

In this paper, we have demonstrated the potential of wavelets with composite dilations to derive and implement directional multiscale representations which are specifically designed to deal with edges and other anisotropic features with high efficiency. A new discrete method for decomposing images using this approach 
was devised which follows directly from the generating structure and is much more flexible and sophisticated than previous design concepts. In fact, this new method is able to produce very sophisticate geometrical decompositions of the frequency plane such as the new hyperbolic decomposition. Our new discrete transforms even improve upon the original implementation of the discrete shearlet transform, whose advantages in denoising and other imaging applications have been established in previous works. The numerical demonstrations included in this paper show that our new discrete transforms perform consistently better than similar directional multiscale transforms in problems of image denoising and enhancement.

Acknowledgments. D.L. acknowledges partial support from NSF grants DMS 1008900 and DMS 1005799 and NHARP grant 003652-0136-2009.

\section{REFERENCES}

[1] R. H. Bamberger and M. J. T. Smith, A filter bank for directional decomposition of images: theory and design, IEEE Trans. Signal Process., 40(2), pp. 882-893, 1992.

[2] C. A. Berenstein, A. Yger, and B. A. Taylor, Sur quelques formules explicites de deconvolution, Journal of Optics (Paris), 14, pp. 75-82, 1983.

[3] C. A. Berenstein, and A. Yger, Le problème de la déconvolution, J. Funct. Anal., pp. 113-160, 1983.

[4] J. D. Blanchard, Minimally supported frequency composite dilation wavelets, J. Fourier Anal. Appl., 15, pp. 796-815, 2009.

[5] J. D. Blanchard, Minimally Supported Frequency Composite Dilation Parseval Frame Wavelets, J. Geom. Anal., 19, pp. 19-35, 2009.

[6] J. D. Blanchard and I. A. Krishtal, Matricial filters and crystallographic composite dilation wavelets, Math. Comp., 81 pp. 905-922, 2012.

[7] E. J. Candès, L. Demanet, D. L. Donoho and L. Ying. Fast discrete curvelet transforms, Multiscale Model. Simul., 5, pp. 861-899, 2006. 
[8] E. J. Candès and D. L. Donoho, New tight frames of curvelets and optimal representations of objects with piecewise $C^{2}$ singularities, Comm. Pure and Appl. Math., 56, pp. 216-266, 2004.

[9] S. G. Chang, B. Yu, and M. Vetterli, Spatially adaptive wavelet thresholding with context modeling for image denoising, IEEE Trans. on Imag. Processing, 9(9), pp. 1522-1531, 2000.

[10] J. Chung, G. R. Easley, and D. P. O'Leary, Windowed Spectral Regularization of Inverse Problems, SIAM J. Sci. Comput., 33(6), pp. 3175-3200, 2012.

[11] F. Colonna, and G. R. Easley, The multichannel deconvolution problem: a discrete analysis, J. Fourier Anal. Appl. 10, pp. 351-376, 2004.

[12] A. L. Cunha, J. Zhou, M. N. Do, The nonsubsampled contourlet transform: Theory, design, and applications, IEEE Trans. Image Processing, 15, pp. 3089-3101, 2006.

[13] M. N. Do and M. Vetterli, The contourlet transform: an efficient directional multiresolution image representation, IEEE Trans. Image Process., 14(12), pp. 2091-2106, 2005.

[14] S. Durand, Orthonormal bases of non-separable wavelets with sharp directions, Proceedings of IEEE Int. Conf. on Image Proc., 2005.

[15] S. Durand, M-band filtering and non-redundant directional wavelets, Appl. Comput. Harmon. Anal., 22(1), pp. 124-139, 2007.

[16] G. Easley, D. Labate, Critically sampled wavelets with composite dilations, IEEE Trans. Image Process., 21(2), pp. 550-561, 2012.

[17] G. Easley, D. Labate and W. Lim, Sparse Directional Image Representations using the Discrete Shearlet Transform, Appl. Comput. Harmon. Anal., 25, pp. 25-46, 2008.

[18] G. R. Easley, and D. F. Walnut, Local multichannel deconvolution, J. Math. Imaging Vision, 18, pp. 69-80, 2003.

[19] K. Egan, Y-T Tseng, N. Holzschuch, F. Durand, R. Ramamorthi, Frequency Analysis and Sheared Reconstruction for Rendering Motion Blur, ACM Transactions on Graphics, 28(3), pp. 1-13, 2009.

[20] R. Eslami and H. Radha, New image transforms using hybrid wavelets and directional filter banks: Analysis and design, Proc. IEEE Int. Conf. Image Process. ICIP2005. Genova, Italy, 2005. 
[21] R. Eslami and H. Radha, Regular hybrid wavelets and directional filter banks: Extensions and applications, Proc. IEEE Int. Conf. Image Process. ICIP2006. Atlanta, GA, 2006.

[22] R. Eslami and H. Radha, A new family of nonredundant transforms using hybrid wavelets and directional filter banks, IEEE Trans. Image Process., 16(4), pp. 1152-1167, 2007.

[23] W. T. Freeman and E. H. Adelson, The design and use of steerable filters, IEEE Trans. Patt. Anal. Mach. Intell., 9, pp. 891-906, 1991.

[24] K. Guo and D. Labate, Optimally sparse multidimensional representation using shearlets, SIAM J. Math. Anal., 9, pp. 298-318, 2007.

[25] K. Guo, and D. Labate, Optimally Sparse 3D Approximations using Shearlet Representations, Electronic Research Announcements in Mathematical Sciences, 17 pp. 126-138, 2010.

[26] K. Guo, D. Labate, W.-Q. Lim, D. Labate, G. Weiss, and E. Wilson, Wavelets with composite dilations, Electron. Res. Announc. Amer. Math. Soc., 10, pp. 78-87, 2004.

[27] K. Guo, D. Labate, W.-Q. Lim, D. Labate, G. Weiss, and E. Wilson, Wavelets with composite dilations and their MRA properties, Appl. Comput. Harmon. Anal., 20, pp. 220-236, 2006.

[28] K. Guo, D. Labate, W.-Q. Lim, D. Labate, G. Weiss, and E. Wilson, The theory of wavelets with composite dilations in: Harmonic Analysis and Applications, C. Heil (ed.), pp. 231-249, Birkhäuser, Boston, MA, 2006.

[29] G. Harikumar, and Y. Bresler, FIR perfect signal reconstruction from multiple convolutions: minimum deconvolver orders, IEEE Trans. Sign. Proc. 46, pp. 215-218, 1998.

[30] J. Lu, D. M. Healy, Jr., Contrast enhancement via multi-scale gradient transformation, in Wavelet Applications, Proceedings of SPIE, Orlando, FL, April 5-8, 1994.

[31] S. Higaki, S. Kyochi, Y. Tanaka, and M. Ikehara, A novel design of critically sampled contourlet transform and its application to image coding, Proc. IEEE Int. Conf. Image Process. ICIP2008. San Diego, CA, Oct. 2008.

[32] I. Kryshtal, B. Robinson, G. Weiss, and E. Wilson, Compactly supported wavelets with composite dilations, J. Geom. Anal. 17, pp. 87-96, 2006.

[33] G. Kutyniok and W. Lim, Compactly supported shearlets are optimally sparse, J. Approx. Theory, 163, pp. 1564-1589, 2011. 
[34] D. Labate, W. Lim, G. Kutyniok, and G. Weiss, Sparse multidimensional representation using shearlets, Wavelets XI (San Diego, CA, 2005), pp. 254-262, SPIE Proc., 5914, SPIE, Bellingham, WA, 2005.

[35] A. F. Laine and X. Zong, A multiscale sub-octave wavelet transform for de-noising and enhancement, in Wavelet Applications, Proceedings of SPIE, Denver, CO, August 6-9, 1996, 2825, pp. 238-249, 1996.

[36] Y. Lu and M. N. Do, The finer directional wavelet transform, Proceedings of IEEE International Conference on Acoustics, Speech, and Signal Processing (ICASSP), Philadelphia, March 2005.

[37] S. Mallat, A Wavelet Tour of Signal Processing, Academic Press, San Diego, 1998.

[38] T. T. Nguyen and S. Oraintara, Multiresolution direction filterbanks: Theory, design, and applications, IEEE Trans. Signal Process., 53(10), pp. 3895-3905, 2005.

[39] P. Schiske, Zur frage der bildrekonstruktion durch fokusreihen, Proc. Eur. Reg. Conf. Electron. Microsc. 4th, pp. 1-145, 1968.

[40] P. Schiske, Image processing using additional statistical information about the object. In P. W. Hawkes, ed., Image Processing and Computer Aided Design in Electron Optics. Academic Press, New York, 1973.

[41] E. P. Simoncelli and E. H. Adelson, Non-separable extensions of quadrature mirror filters to multiple dimensions, Proceedings of the IEEE, 78(4), pp. 652-664, 1990.

[42] J. L. Starck, E. J. Candès, and D. L. Donoho, The curvelet transform for image denoising, IEEE Trans Image Process., 11(6), pp. 670-684, 2002.

[43] J. L. Starck, F. Murtagh, E. Candes, and D. L. Donoho, Gray and Color Image Contrast Enhancement by the Curvelet Transform, IEEE Trans. on Imag. Processing, 12(6), pp. 706$717,2003$.

[44] J. L. Starck, E. J. Candes, and D. L. Donoho, The Curvelet Transform for image denoising, IEEE Trans. on Imag. Processing, 11(6), pp. 670-684, 2002.

[45] Y. Tanaka, M. Ikehara, and T. Q. Nguyen, Multiresolution image representation using combined 2D and 1D directional filter banks, IEEE Trans. Image Process., 18(2), pp. 269-280, 2009. 
[46] Z. Wang and A. C. Bovik, A universal image quality index, IEEE Signal Processing Letters, 9, pp. 81-84, 2002.

[47] S. Yi, D. Labate, G. R. Easley, and H. Krim, A Shearlet approach to Edge Analysis and Detection, IEEE Trans. Image Process 18(5), pp. 929-941, (2009).

[48] J. Zhou, and M. N. Do, Multidimensional multichannel FIR deconvolution using Gröbner bases, IEEE Trans. Image Proc. 15, pp. 2998-3007, 2006.

\section{APPENDix}

Below is a Matlab-based pseudo-code to generate the hyperbolic composite wavelet filters restricted to the fourth quadrant. The complete filters are found by adding the appropriate flip with zero padding. $N_{0}$ is the quadrant size of the image and $k \geq 2$ is a multiplier to determine the number of sequence elements. $\mathrm{Hr}$ and $\mathrm{Ht}$ denote the window functions for the radial and time parameters. 


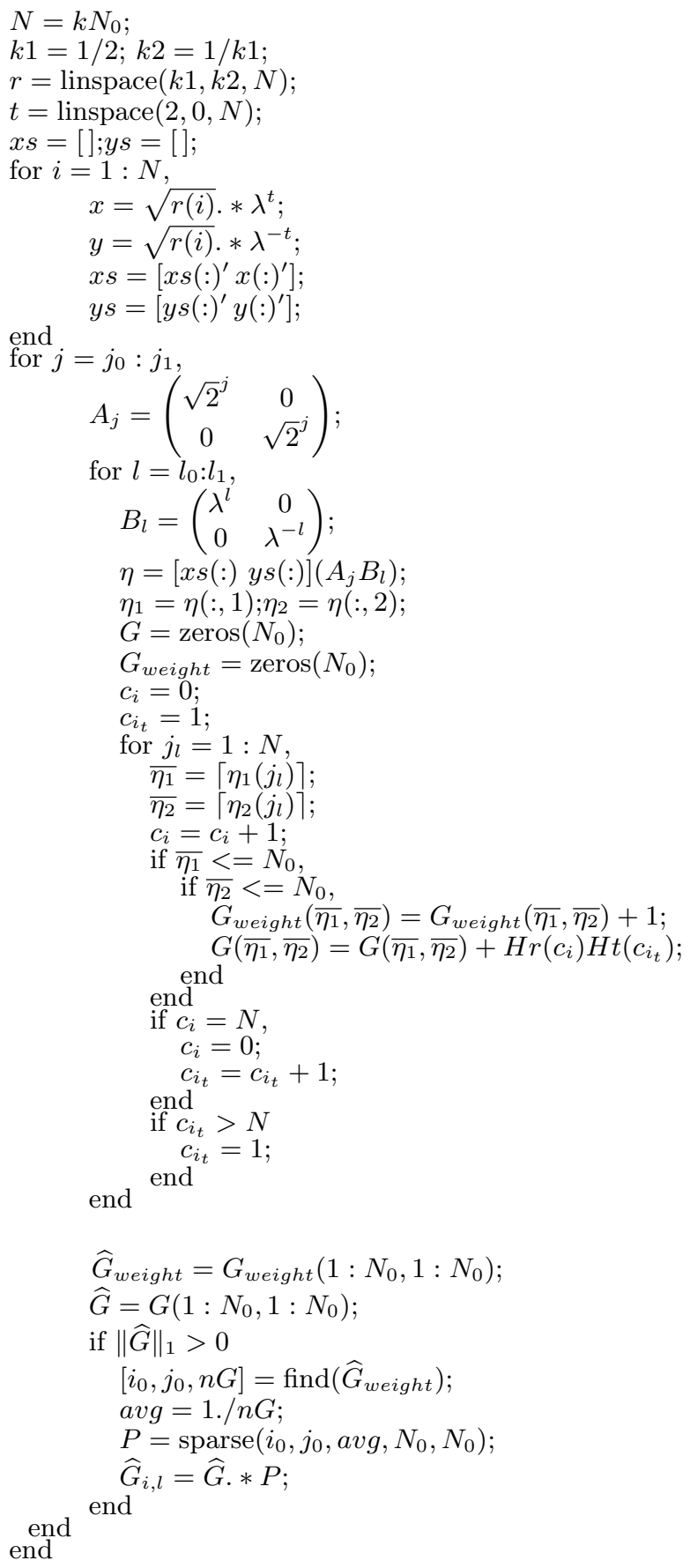


Department of Mathematics, University of Houston, Houston, TX 77204, USA

E-mail address: dlabate@math.uh.edu

University of Maryland, College Park, MD 20742

E-mail address: pvishalm@umiacs.umd.edu 